\title{
Pacific
}

Journal of

Mathematics

\section{COXETER GROUPS, IMAGINARY CONES AND DOMINANCE}

XIANG FU 


\title{
COXETER GROUPS, IMAGINARY CONES AND DOMINANCE
}

\author{
XIANG FU
}

\begin{abstract}
Brink and Howlett [Math. Ann. 296:1 (1993), 179-190] have introduced a partial ordering, called dominance, on the root systems of Coxeter groups in their proof that all finitely generated Coxeter groups are automatic. Edgar (Ph.D. thesis, 2009), in an investigation of various regularity properties of Coxeter groups, studied a function on the reflections of such groups, called $\infty$-height. Here we show that these two concepts are closely related to each other. We also give applications of dominance to the study of imaginary cones of Coxeter groups.
\end{abstract}

\section{Introduction}

In this paper we attempt to extend the understanding of a partial ordering (called dominance) defined on the root system of an arbitrary Coxeter group. The dominance ordering was introduced in [Brink and Howlett 1993] (where it was used to prove the automaticity of all finitely generated Coxeter groups). Dominance ordering was further studied in [Brink 1998; Krammer 1994; 2009], and it has only been recently examined again, in [Dyer 2012] (in connection with the representation theory of Coxeter groups) and in [Edgar 2009; Fu 2012]. The present paper is a short addition to the last two references, and it could serve as a building block in the general knowledge of dominance ordering and of the combinatorics and geometry of Coxeter groups in general.

More specifically, this paper has the following two objectives: (1) investigating the connection between the dominance ordering on the root system of an arbitrary Coxeter groups $W$ and a specific function (called $\infty$-height) defined on the set of reflections of $W$; and (2) exploring the applications of the dominance ordering to the imaginary cone of $W$ (as defined by Kac).

The paper is organized into three sections. The first introduces background material: root bases, Coxeter data, and root systems are defined in the context of the paper, and some basic properties of Coxeter groups are recalled for later use in the

The work presented in this paper was completed when the author was supported by the Australian Research Council Discovery Project Invariant theory, cellularity and geometry, no. DP0772870.

MSC2010: primary 20F55, 20F65; secondary 20F10.

Keywords: Coxeter groups, root systems, Tits cone, dominance. 
paper (most of them can be found in [Howlett 1996]). Here we follow the definition used in [Krammer 1994], which gives a slight variant of the classical notion of root systems, particularly adapted when working with arbitrary (not necessarily crystallographic) Coxeter groups. Furthermore, this framework allows easy passing to reflection subgroups. Indeed, we recall the fundamental property [Dyer 1987, Theorem 1.8] that the reflection subgroups of a Coxeter group are themselves Coxeter groups, and this particular framework allows us to apply all the definitions and properties to the reflection subgroups and not only to the overgroup.

In the second section, the first main theorem (giving the connection between $\infty$-height and dominance order) is stated and proved. All results are related to an arbitrary Coxeter datum, implying the data of a root system $\Phi$, its associated Coxeter group $W$, and the set $T$ of all reflections of $W$ (consisting of all the $W$-conjugates of the Coxeter generators). The main objects of study are:

- The dominance order on $\Phi$ (Definition 3.1). Given $x, y \in \Phi$, we say $x$ dominates $y$ if whenever $w \in W$ such that $w x \in \Phi^{-}$then $w y \in \Phi^{-}$too (where $\Phi^{-}$denotes the set of negative roots).

- The $\infty$-height function on $T$. This is a variant of the usual (standard) height function of a reflection $t \in T$, namely, the minimal length of an element of $W$ that maps $\alpha_{t}$ (the unique positive root associated to $t$ ) to an element of the root basis. Adhering to the general framework of this paper, our definition of the height function applies to all reflection subgroups of $W$. It is easy to check (Lemma 3.13) that the height of $t$ is equal to the sum of the heights of $t$ relative to each maximal (with respect to inclusion) dihedral reflection subgroup containing $t$. The $\infty$-height of $t$ is then defined as a subsum of this sum, taking into account only those subgroups which are infinite (Definition 3.8).

We then show that these two concepts are closely related in the following way. The canonical bijection $t \leftrightarrow \alpha_{t}$, between $T$ and $\Phi^{+}$(the set of positive roots), restricts to a bijection between (for any $n \in \mathbb{N}$ )

- the set $T_{n}$ of all reflections whose $\infty$-height is $n$, and

- the set $D_{n}$ of all positive roots which strictly dominate exactly $n$ other positive roots.

The proof of this fact (Theorem 3.15) relies on a study of dihedral reflection subgroups. We have previously studied the partition $\left(D_{n}\right)_{n \in \mathbb{N}}$ of $\Phi^{+}$in [Fu 2012]; in particular, we showed there that each $D_{n}$ is finite and we gave an upper bound for its cardinality. Together with Theorem 3.15, this allows us to deduce here some further information on the combinatorics of the $T_{n}$ 's (Corollary 3.23).

The final section explores the relation between the dominance order and the imaginary cone of a Coxeter group. The concept of imaginary cone was introduced 
in [Kac 1990] to study the imaginary roots of Kac-Moody Lie algebras, and was later generalized to Coxeter groups by Hée [1990; 1993] and Dyer [2012]. It is defined as the subset of the dual of the Tits cone (denoted by $U^{*}$ here) consisting of elements $v \in U^{*}$ such that $(v, \alpha)>0$ for only finitely many $\alpha \in \Phi^{+}$(where ( , ) denotes the bilinear form associated to the Coxeter datum). The main results (Theorem 4.13 and Corollary 4.15) of this section state the following property: whenever $x, y \in \Phi$, then $x$ dominates $y$ if and only if $x-y$ lies in the imaginary cone. One direction of this property was first suggested to us by Howlett [private communication], and it is a special case of a result obtained independently (but earlier) by Dyer. We are deeply indebted to both of them for helpful discussions inspiring us to study the imaginary cone. We would also like to thank the referee of this paper for many valuable suggestions, especially those resulting in Corollary 4.15. To close this section, we include an alternative definition for the imaginary cone in the case where $W$ is finitely generated.

\section{Background material}

Definition 2.1 [Krammer 1994]. Suppose that $V$ is a vector space over $\mathbb{R}$. Let ( , ) be a bilinear form on $V$ and let $\Delta$ be a subset of $V$. Then $\Delta$ is called a root basis if the following conditions are satisfied:

(C1) $(a, a)=1$ for all $a \in \Delta$, and for distinct elements $a, b \in \Delta$, either $(a, b)=$ $-\cos \left(\pi / m_{a b}\right)$ for some integer $m_{a b}=m_{b a} \geq 2$, or else $(a, b) \leq-1$ (in which case we define $\left.m_{a b}=m_{b a}=\infty\right)$.

(C2) $0 \notin \mathrm{PLC}(\Pi)$, where the the positive linear cone of a set $A$ is defined by

$$
\operatorname{PLC}(A)=\left\{\sum_{a \in A} \lambda_{a} a \mid \lambda_{a} \geq 0 \text { for all } a \in A \text { and } \lambda_{a^{\prime}}>0 \text { for some } a^{\prime} \in A\right\} .
$$

If $\Delta$ is a root basis, then we call the triple $\mathscr{b}=(V, \Delta,()$,$) a Coxeter datum.$ Throughout this paper we fix a particular Coxeter datum $\mathscr{C}$. We stress that our definition of a root basis is not the most classical one of [Bourbaki 1968] or even [Humphreys 1990]: the root system (see Definition 2.5) arising from our definition of a root basis is not necessarily crystallographic (indeed, the bilinear form can take values less than -1 ), and the root basis is not assumed to be linearly independent (this allows us to transmit easily the definitions and properties of a Coxeter group to its reflection subgroups; indeed, the requirements in our definition of a root basis of a Coxeter group are identical to those in the characterization of the equivalent of a root basis in any reflection subgroup). Observe that (C1) implies that $a \notin \operatorname{PLC}(\Delta \backslash\{a\})$ if $a \in \Delta$, and (C1) and (C2) together imply that $\{a, b, c\}$ is linearly independent for all distinct $a, b, c \in \Delta$. Note also that (C2) is equivalent to the requirement that zero does not lie in the convex hull of $\Delta$. 
For each $a \in \Delta$, define $\rho_{a} \in \mathrm{GL}(V)$ by the rule $\rho_{a} x=x-2(x, a) a$ for all $x \in V$. Observe that $\rho_{a}$ is a reflection, and $\rho_{a} a=-a$. We summarize a few useful results:

Proposition 2.2 [Howlett 1996, Lecture 1, propositions on pp. 2-3]. (i) Suppose that $a, b \in \Delta$ are distinct such that $m_{a b} \neq \infty$. Set $\theta=\pi / m_{a b}$. Then

$$
\left(\rho_{a} \rho_{b}\right)^{i} a=\frac{\sin (2 i+1) \theta}{\sin \theta} a+\frac{\sin 2 i \theta}{\sin \theta} b
$$

for each integer $i$, and in particular, $\rho_{a} \rho_{b}$ has order $m_{a b}$ in $\operatorname{GL}(V)$.

(ii) Suppose that $a, b \in \Delta$ are distinct such that $m_{a b}=\infty$. Set $\theta=\cosh ^{-1}(-(a, b))$. Then

$$
\left(\rho_{a} \rho_{b}\right)^{i} a=\left\{\begin{array}{cc}
\frac{\sinh (2 i+1) \theta}{\sinh \theta} a+\frac{\sinh 2 i \theta}{\sinh \theta} b & \text { if } \theta \neq 0, \\
(2 i+1) a+2 i b & \text { if } \theta=0,
\end{array}\right.
$$

for each integer $i$, and in particular, $\rho_{a} \rho_{b}$ has infinite order in $\mathrm{GL}(V)$.

Let $G_{\mathscr{b}}$ be the subgroup of GL(V) generated by $\left\{\rho_{a} \mid a \in \Delta\right\}$. Suppose that $(W, S)$ is a Coxeter system in the sense of [Hiller 1982] or [Humphreys 1990] with $S=\left\{r_{a} \mid a \in \Delta\right\}$ being a set of involutions generating $W$ subject only to the condition that the order of $r_{a} r_{b}$ is $m_{a b}$ for all $a, b \in \Delta$ with $m_{a b} \neq \infty$. Then Proposition 2.2 yields that there exists a group homomorphism $\phi_{\mathfrak{C}}: W \rightarrow G_{\mathfrak{C}}$ satisfying $\phi_{\mathfrak{C}}\left(r_{a}\right)=\rho_{a}$ for all $a \in \Delta$. This homomorphism, together with the $G_{\mathscr{C}}$-action on $V$, gives rise to a $W$-action on $V$ : for each $w \in W$ and $x \in V$, define $w x \in V$ by $w x=\phi_{\mathscr{C}}(w) x$. It can be easily checked that this $W$-action preserves (, ). Denote the length function of $W$ with respect to $S$ by $\ell$, and call an expression $w=r_{1} r_{2} \cdots r_{n}$ (where $w \in W$ and $r_{i} \in S$ ) reduced if $\ell(w)=n$. The following is a useful result:

Proposition 2.3 [Howlett 1996, Lecture 1, theorem, p. 4]. Let $G_{\mathfrak{b}}, W, S$ and $\ell$ be as above, and let $w \in W$ and $a \in \Delta$. If $\ell\left(w r_{a}\right) \geq \ell(w)$, then $w a \in \operatorname{PLC}(\Delta)$.

An immediate consequence of the proposition is the following important fact: Corollary 2.4 [Howlett 1996, Lecture 1, corollary, p. 5]. Let $G_{\mathscr{b}}, W, S$ and $\phi_{\mathscr{C}}$ be as above. Then $\phi_{\mathfrak{b}}: W \rightarrow G_{\mathfrak{C}}$ is an isomorphism.

In particular, the corollary yields that $\left(G_{\mathfrak{b}},\left\{\rho_{a} \mid a \in \Delta\right\}\right)$ is a Coxeter system isomorphic to $(W, S)$. We call $(W, S)$ the abstract Coxeter system associated to the Coxeter datum $\mathscr{C}$, and we call $W$ a Coxeter group of rank \#S (where \# denotes cardinality).

Definition 2.5. The root system of $W$ in $V$ is the set

$$
\Phi=\{w a \mid w \in W \text { and } a \in \Delta\} .
$$

The set $\Phi^{+}=\Phi \cap \operatorname{PLC}(\Delta)$ is called the set of positive roots, and the set $\Phi^{-}=-\Phi^{+}$ is called the set of negative roots. 
From Proposition 2.3 we may readily deduce that:

Proposition 2.6 [Howlett 1996, Lecture 3, corollary on p. 11, proposition on p. 10 and lemma on p. 4]. (i) Let $w \in W$ and $a \in \Delta$. Then

$$
\ell\left(w r_{a}\right)= \begin{cases}\ell(w)-1 & \text { if } w a \in \Phi^{-}, \\ \ell(w)+1 & \text { if } w a \in \Phi^{+} .\end{cases}
$$

(ii) $\Phi=\Phi^{+} \uplus \Phi^{-}$, where $\uplus$ denotes disjoint union.

(iii) $W$ is finite if and only if $\Phi$ is finite.

Define $T=\bigcup_{w \in W} w S w^{-1}$. We call $T$ the set of reflections in $W$. For each $x \in$ $\Phi$, let $\rho_{x} \in \mathrm{GL}(V)$ be defined by the rule $\rho_{x}(v)=v-2(v, x) x$ for all $v \in V$. Since $x \in \Phi$, it follows that $x=w a$ for some $w \in W$ and $a \in \Delta$. Direct calculations yield that $\rho_{x}=\left(\phi_{\mathscr{C}}(w)\right) \rho_{a}\left(\phi_{\mathscr{C}}(w)\right)^{-1} \in G_{\mathscr{C}}$. Now let $r_{x} \in W$ be such that $\phi_{\mathscr{C}}\left(r_{x}\right)=\rho_{x}$. Then $r_{x}=w r_{a} w^{-1} \in T$, and we call $r_{x}$ the reflection corresponding to $x$. It is readily checked that $r_{x}=r_{-x}$ for all $x \in \Phi$ and $T=\left\{r_{x} \mid x \in \Phi\right\}$. For each $t \in T$ we let $\alpha_{t}$ be the unique positive root with the property that $r_{\alpha_{t}}=t$. It is also easily checked that there is a bijection $\psi: T \rightarrow \Phi^{+}$given by $\psi(t)=\alpha_{t}$, and we call $\psi$ the canonical bijection.

For each $x \in \Phi^{+}$, as in [Brink and Howlett 1993], we define the depth of $x$ relative to $S$ to be $\min \left\{\ell(w) \mid w \in W\right.$ and $\left.w x \in \Phi^{-}\right\}$, and we denote it by $\operatorname{dp}(x)$. The following lemma gives some basic properties of depth:

Lemma 2.7 [Brink and Howlett 1993; Brink 1994; Saunders 1991]. (i) Let $\alpha \in \Phi^{+}$. Then $\operatorname{dp}(\alpha)=\frac{1}{2}\left(\ell\left(r_{\alpha}\right)+1\right)$.

(ii) Let $r \in S$ and $\alpha \in \Phi^{+} \backslash\left\{\alpha_{r}\right\}$. Then

$$
\operatorname{dp}(r \alpha)= \begin{cases}\operatorname{dp}(\alpha)-1 & \text { if }\left(\alpha, \alpha_{r}\right)>0 \\ \operatorname{dp}(\alpha) & \text { if }\left(\alpha, \alpha_{r}\right)=0 \\ \operatorname{dp}(\alpha)+1 & \text { if }\left(\alpha, \alpha_{r}\right)<0\end{cases}
$$

Proof. (i) See [Brink 1994, Corollary 2.7]. This is also a special case of [Fu 2010, Lemma 1.3.19].

(ii) See [Brink and Howlett 1993, Lemma 1.7].

Remark 2.8. Part (i) of Lemma 2.7 is equivalent to the property that any reflection in a Coxeter group has a palindromic expression which is reduced, and this was indeed noted in [Saunders 1991, Proposition 4.3].

Define functions $N: W \rightarrow \mathscr{P}\left(\Phi^{+}\right)$and $\bar{N}: W \rightarrow \mathscr{P}(T)$ (where $\mathscr{P}$ denotes the power set) by setting

$$
\begin{aligned}
& N(w)=\left\{x \in \Phi^{+} \mid w x \in \Phi^{-}\right\}, \\
& \bar{N}(w)=\{t \in T \mid \ell(w t)<\ell(w)\},
\end{aligned}
$$


for all $w \in W$. We call $\bar{N}$ the reflection cocycle of $W$ (sometimes $\bar{N}(w)$ is also called the right descent set of $w$ ). Standard arguments such as those in [Humphreys 1990, Section 5.6] yield that, for each $w \in W$,

$$
\ell(w)=\# N(w)
$$

and

$$
\bar{N}(w)=\left\{r_{x} \mid x \in N(w)\right\} .
$$

In particular, $N\left(r_{a}\right)=\{a\}$ for $a \in \Delta$. Moreover, $\ell\left(w v^{-1}\right)+\ell(v)=\ell(w)$ for some $w, v \in W$ if and only if $N(v) \subseteq N(w)$.

A subgroup $W^{\prime}$ of $W$ is a reflection subgroup of $W$ if it is generated by the reflections contained in it: $W^{\prime}=\left\langle W^{\prime} \cap T\right\rangle$. For any reflection subgroup $W^{\prime}$ of $W$, let

$$
S\left(W^{\prime}\right)=\left\{t \in T \mid \bar{N}(t) \cap W^{\prime}=\{t\}\right\} \quad \text { and } \quad \Delta\left(W^{\prime}\right)=\left\{x \in \Phi^{+} \mid r_{x} \in S\left(W^{\prime}\right)\right\} .
$$

It was shown by Dyer [1990] and Deodhar [1982] that $\left(W^{\prime}, S\left(W^{\prime}\right)\right)$ forms a Coxeter system:

Theorem 2.9 (Dyer). (i) Suppose that $W^{\prime}$ is an arbitrary reflection subgroup of $W$. Then $\left(W^{\prime}, S\left(W^{\prime}\right)\right)$ forms a Coxeter system. Moreover, $W^{\prime} \cap T=$ $\bigcup_{w \in W^{\prime}} w S\left(W^{\prime}\right) w^{-1}$.

(ii) Suppose that $W^{\prime}$ is a reflection subgroup of $W$, and suppose that $a, b \in \Delta\left(W^{\prime}\right)$ are distinct. Then

$$
(a, b) \in\{-\cos (\pi / n) \mid n \in \mathbb{N} \text { and } n \geq 2\} \cup(-\infty,-1] .
$$

Conversely, if $\Delta^{\prime}$ is a subset of $\Phi^{+}$satisfying the condition that

$$
(a, b) \in\{-\cos (\pi / n) \mid n \in \mathbb{N} \text { and } n \geq 2\} \cup(-\infty,-1]
$$

for all $a, b \in \Delta^{\prime}$ with $a \neq b$, then $\Delta^{\prime}=\Delta\left(W^{\prime}\right)$ for some reflection subgroup $W^{\prime}$ of $W$. In fact, $W^{\prime}=\left\langle\left\{r_{a} \mid a \in \Delta^{\prime}\right\}\right\rangle$.

Proof. (i) See [Dyer 1990, Theorem 3.3].

(ii) See [Dyer 1990, Theorem 4.4].

Let $(,)^{\prime}$ be the restriction of $($,$) to the subspace \operatorname{span}\left(\Delta\left(W^{\prime}\right)\right)$. Then $\mathscr{C}^{\prime}=$ $\left(\operatorname{span}\left(\Delta\left(W^{\prime}\right)\right), \Delta\left(W^{\prime}\right),(,)^{\prime}\right)$ is a Coxeter datum with $\left(W^{\prime}, S\left(W^{\prime}\right)\right)$ being the associated abstract Coxeter system. Thus the notion of a root system applies to $\mathscr{C}^{\prime}$. We let $\Phi\left(W^{\prime}\right), \Phi^{+}\left(W^{\prime}\right)$ and $\Phi^{-}\left(W^{\prime}\right)$ be, respectively, the set of roots, positive roots and negative roots for the datum $\mathscr{C}^{\prime}$. Then $\Phi\left(W^{\prime}\right)=W^{\prime} \Delta\left(W^{\prime}\right)$, and Theorem 2.9(i) yields that $\Phi\left(W^{\prime}\right)=\left\{x \in \Phi \mid r_{x} \in W^{\prime}\right\}$. Furthermore, we have $\Phi^{+}\left(W^{\prime}\right)=\Phi\left(W^{\prime}\right) \cap \operatorname{PLC}\left(\Delta\left(W^{\prime}\right)\right)$ and $\Phi^{-}\left(W^{\prime}\right)=-\Phi^{+}\left(W^{\prime}\right)$. We call $S\left(W^{\prime}\right)$ the set of canonical generators of $W^{\prime}$, and we call $\Delta\left(W^{\prime}\right)$ the set of canonical roots 
of $\Phi\left(W^{\prime}\right)$. In this paper a reflection subgroup $W^{\prime}$ is called a dihedral reflection subgroup if $\# S\left(W^{\prime}\right)=2$.

A subset $\Phi^{\prime}$ of $\Phi$ is called a root subsystem if $r_{y} x \in \Phi^{\prime}$ whenever $x, y$ are both in $\Phi^{\prime}$. It is easily seen that there is a bijective correspondence between the set of reflection subgroups $W^{\prime}$ of $W$ and the set of root subsystems $\Phi^{\prime}$ of $\Phi$ : $W^{\prime}$ uniquely determines the root subsystem $\Phi\left(W^{\prime}\right)$, and $\Phi^{\prime}$ uniquely determines the reflection subgroup $\left\langle\left\{r_{x} \mid x \in \Phi^{\prime}\right\}\right\rangle$.

The notion of a length function also applies to the Coxeter system $\left(W^{\prime}, S\left(W^{\prime}\right)\right)$, and we let $\ell_{\left(W^{\prime}, S\left(W^{\prime}\right)\right)}: W^{\prime} \rightarrow \mathbb{N}$ be the length function for $\left(W^{\prime}, S\left(W^{\prime}\right)\right)$. If $w \in$ $W^{\prime}$ and $a \in \Delta\left(W^{\prime}\right)$ then applying Proposition 2.6 to the Coxeter datum $\mathscr{C}^{\prime}=$ $\left(\operatorname{span}\left(\Delta\left(W^{\prime}\right)\right), \Delta\left(W^{\prime}\right),(),\right)$ yields

$$
\ell_{\left(W^{\prime}, S\left(W^{\prime}\right)\right)}\left(w r_{a}\right)= \begin{cases}\ell_{\left(W^{\prime}, S\left(W^{\prime}\right)\right)}(w)-1 & \text { if } w a \in \Phi^{-}\left(W^{\prime}\right), \\ \ell_{\left(W^{\prime}, S\left(W^{\prime}\right)\right)}(w)+1 & \text { if } w a \in \Phi^{+}\left(W^{\prime}\right) .\end{cases}
$$

Similarly, the notion of a reflection cocycle also applies to the Coxeter system $\left(W^{\prime}, S\left(W^{\prime}\right)\right)$. Let $\bar{N}_{\left(W^{\prime}, S\left(W^{\prime}\right)\right)}: W \rightarrow \mathscr{P}\left(W^{\prime} \cap T\right)$ denote the reflection cocycle for $\left(W^{\prime}, S\left(W^{\prime}\right)\right)$. Then, for each $w \in W^{\prime}$,

$$
\bar{N}_{\left(W^{\prime}, S\left(W^{\prime}\right)\right)}(w)=\left\{t \in W^{\prime} \cap T \mid \ell_{\left(W^{\prime}, S\left(W^{\prime}\right)\right)}(w t)<\ell_{\left(W^{\prime}, S\left(W^{\prime}\right)\right)}(w)\right\},
$$

and we define $N_{\left(W^{\prime}, S\left(W^{\prime}\right)\right)}(w)=\left\{x \in \Phi^{+}\left(W^{\prime}\right) \mid w x \in \Phi^{-}\left(W^{\prime}\right)\right\}$ for each $w \in W^{\prime}$. It is shown in [Dyer 1987] that $\bar{N}_{\left(W^{\prime}, S\left(W^{\prime}\right)\right)}(w)=\bar{N}(w) \cap W^{\prime}$ for an arbitrary reflection subgroup $W^{\prime}$ of $W$. Furthermore, it is readily seen that the canonical bijection $\psi$ restricts to a bijection $\psi^{\prime}: T \cap W^{\prime} \rightarrow \Phi^{+}\left(W^{\prime}\right)$ given by $\psi^{\prime}(t)=\alpha_{t}$. For $w \in W^{\prime}$, applying (2-1) to the Coxeter datum $\mathscr{C}^{\prime}=\left(\operatorname{span}\left(\Delta\left(W^{\prime}\right), \Delta\left(W^{\prime}\right),(,)^{\prime}\right)\right.$ yields that

$$
\ell_{\left(W^{\prime}, S\left(W^{\prime}\right)\right)}(w)=\# N_{\left(W^{\prime}, S\left(W^{\prime}\right)\right)}(w)
$$

Furthermore, $\ell_{\left(W^{\prime}, S\left(W^{\prime}\right)\right)}\left(w v^{-1}\right)+\ell_{\left(W^{\prime}, S\left(W^{\prime}\right)\right)}(v)=\ell_{\left(W^{\prime}, S\left(W^{\prime}\right)\right)}(w)$ for some $w$, $v \in W^{\prime}$ precisely when $N_{\left(W^{\prime}, S\left(W^{\prime}\right)\right)}(v) \subseteq N_{\left(W^{\prime}, S\left(W^{\prime}\right)\right)}(w)$.

For a Coxeter datum $\mathscr{C}=(V, \Delta,()$,$) , since \Delta$ may be linearly dependent, the expression of a root in $\Phi$ as a linear combination of elements of $\Delta$ may not be unique. Thus the concept of the coefficient of an element of $\Delta$ in any given root in $\Phi$ is potentially ambiguous. We close this section by specifying a canonical way of expressing a root in $\Phi$ as a linear combination of elements from $\Delta$. This canonical expression follows from a standard construction similar to that considered in [Howlett et al. 1997, Proposition 2.9].

Given a Coxeter datum $\mathscr{C}=(V, \Delta,()$,$) , let E$ be a vector space over $\mathbb{R}$ with basis $\Delta_{E}=\left\{e_{a} \mid a \in \Delta\right\}$ in bijective correspondence with $\Delta$, and let (, $)_{E}$ be the 
unique bilinear form on $E$ satisfying

$$
\left(e_{a}, e_{b}\right)_{E}=(a, b) \text { for all } a, b \in \Delta .
$$

Then $\mathscr{C}_{E}=\left(E, \Delta_{E},(,)_{E}\right)$ is a Coxeter datum. Moreover, $\mathscr{C}_{E}$ and $\mathscr{C}$ are associated to the same abstract Coxeter system $(W, S)$; indeed, Corollary 2.4 yields that the abstract Coxeter group $W$ is isomorphic to both $G_{\mathscr{C}}=\left\langle\left\{\rho_{a} \mid a \in \Delta\right\}\right\rangle$ and $G_{\mathscr{C}_{E}}=\left\langle\left\{\rho_{e_{a}} \mid a \in \Delta\right\}\right\rangle$. Furthermore, $W$ acts faithfully on $E$ via $r_{a} y=\rho_{e_{a}} y$ for all $a \in \Delta$ and $y \in E$.

Let $f: E \rightarrow V$ be the unique linear map satisfying $f\left(e_{a}\right)=a$ for all $a \in \Delta$. It is readily checked that $(f(x), f(y))=(x, y)_{E}$ for all $x, y \in E$. Now, for all $a \in \Delta$ and $y \in E$,

$$
\begin{aligned}
r_{a}(f(y)) & =\rho_{a}(f(y))=f(y)-2(f(y), a) a=f(y)-2\left(f(y), f\left(e_{a}\right)\right) f\left(e_{a}\right) \\
& =f\left(y-2\left(y, e_{a}\right)_{E} e_{a}\right)=f\left(r_{a} y\right) .
\end{aligned}
$$

Then it follows that $w(f(y))=f(w y)$ for all $w \in W$ and all $y \in E$, since $W$ is generated by $\left\{r_{a} \mid a \in \Delta\right\}$. Let $\Phi_{E}$ denote the root system associated to the datum $\mathscr{C}_{E}$. Standard arguments yield that:

Proposition 2.10 [Fu 2012, Proposition 2.1]. The restriction of $f$ defines $a W$ equivariant bijection $\Phi_{E} \leftrightarrow \Phi$.

Since $\Delta_{E}$ is linearly independent, it follows that each root $y \in \Phi_{E}$ can be written uniquely as $y=\sum_{e_{a} \in \Delta_{E}} \lambda_{a} e_{a}$; we write $\lambda_{a}=\operatorname{coeff}_{e_{a}}(y)$ and call it the coefficient of $e_{a}$ in $y$. We use this uniqueness together with the $W$-equivariant bijection $f: \Phi_{E} \leftrightarrow \Phi$ to give a canonical expression of a root in $\Phi$ in terms of $\Delta$ :

Definition 2.11. Suppose that $x \in \Phi$. For each $a \in \Delta$, define the canonical coefficient of $a$ in $x$, written coeff $a_{a}(x)$, by requiring that $\operatorname{coeff}_{a}(x)=\operatorname{coeff}_{e_{a}}\left(f^{-1}(x)\right)$. The support, written $\operatorname{supp}(x)$, is the set of $a \in \Delta$ with $\operatorname{coeff}_{a}(x) \neq 0$.

\section{Dominance, maximal dihedral reflection subgroups and infinity height}

Throughout this section, let $W$ be the abstract Coxeter group associated to the Coxeter datum $\mathscr{C}=(V, \Delta,()$,$) , and let \Phi$ and $T$ be the corresponding root system and the set of reflections, respectively. Recently, in [Edgar 2009], a uniquely determined nonnegative integer, called $\infty$-height, was assigned to each reflection in $W$. (Edgar attributes the concept to Dyer.) Naturally, the set $T$ is then the disjoint union of the sets $T_{0}, T_{1}, T_{2}, \ldots$, where the set $T_{n}$ consists of all the reflections with $\infty$-height equal to $n$.

These $T_{n}$ were used in [Edgar 2009, Chapter 5] to demonstrate nice regularity properties of $W$. They gave rise to a family of modules in the generic IwahoriHecke algebra associated to $W$, and in turn, these modules were used by Dyer 
(unpublished) to prove a weak form of Lusztig's conjecture on the boundedness of the a-function. Dyer also showed that if $W$ is of finite rank, then there are finitely many reflections in $T_{n}$ for each $n$.

In this section we prove that for an arbitrary reflection $t \in T$ whose $\infty$-height equals $n$, the corresponding positive root $\alpha_{t}$ dominates precisely $n$ other positive roots. This observation will then establish a bijection between the set of all reflections in $W$ with $\infty$-height equal to $n$ and the set of all positive roots that dominate precisely $n$ other positive roots. Recent results on dominance obtained in [Fu 2012] may then be immediately applied to the $T_{n}$ 's, answering a number of basic questions about these $T_{n}$ 's.

Following [Howlett et al. 1997] and [Björner and Brenti 2005, Section 4.7], we generalize the definition of dominance to the whole of $\Phi$ (whereas in [Brink and Howlett 1993] and [Brink 1998], dominance was only defined on $\Phi^{+}$), and we stress that all the notations are the same as in the previous section.

Definition 3.1. (i) Let $W^{\prime}$ be a reflection subgroup of $W$, and let $x, y \in \Phi\left(W^{\prime}\right)$. Then we say that $x$ dominates $y$ with respect to $W^{\prime}$ if

$$
\left\{w \in W^{\prime} \mid w x \in \Phi^{-}\left(W^{\prime}\right)\right\} \subseteq\left\{w \in W^{\prime} \mid w y \in \Phi^{-}\left(W^{\prime}\right)\right\} .
$$

If $x$ dominates $y$ with respect to $W^{\prime}$ then we write $x \operatorname{dom}_{W^{\prime}} y$.

(ii) Let $W^{\prime}$ be a reflection subgroup of $W$ and let $x \in \Phi^{+}\left(W^{\prime}\right)$. Define

$$
D_{W^{\prime}}(x)=\left\{y \in \Phi^{+}\left(W^{\prime}\right) \mid y \neq x \text { and } x \operatorname{dom}_{W^{\prime}} y\right\} .
$$

If $D_{W^{\prime}}(x)=\varnothing$ we call $x$ elementary with respect to $W^{\prime}$. For each nonnegative integer $n$, define

$$
D_{W^{\prime}, n}=\left\{x \in \Phi^{+}\left(W^{\prime}\right) \mid \# D_{W^{\prime}}(x)=n\right\} .
$$

If $W^{\prime}=W$, we write $D(x)$ for $D_{W^{\prime}}(x)$ and $D_{n}$ for $D_{W^{\prime}, n}$. If $D(x)=\varnothing$ then we call $x$ elementary.

It is readily checked that dominance with respect to any reflection subgroup $W^{\prime}$ of a Coxeter group $W$ is a partial ordering on $\Phi\left(W^{\prime}\right)$. The following lemma summarizes some basic properties of dominance:

Lemma 3.2 [Fu 2012, Lemma 3.2]. (i) Let $x, y \in \Phi^{+}$be arbitrary. Then $x \operatorname{dom}_{W} y$ if and only if $(x, y) \geq 1$ and $\mathrm{dp}(x) \geq \mathrm{dp}(y)$.

(ii) Dominance is $W$-invariant, that is, if $x \operatorname{dom}_{W} y$ then $w x \operatorname{dom}_{W} w y$ for all $w \in W$.

(iii) Let $x, y \in \Phi$ be such that $x \operatorname{dom}_{W} y$. Then $-y \operatorname{dom}_{W}-x$.

(iv) Let $x, y \in \Phi$. Then there is dominance between $x$ and $y$ if and only if $(x, y) \geq 1$. 
Corollary 3.3. Let $x, y \in \Phi$, and let $W^{\prime}$ be an arbitrary reflection subgroup containing both $r_{x}$ and $r_{y}$.

(i) There is dominance with respect to $W^{\prime}$ between $x$ and $y$ if and only if $(x, y)^{\prime} \geq 1$, where $(,)^{\prime}$ is the restriction of $($,$) to the subspace \operatorname{span}\left(\Delta\left(W^{\prime}\right)\right)$.

(ii) $x \operatorname{dom}_{W} y$ if and only if $x \operatorname{dom}_{W^{\prime}} y$.

Proof. (i) This follows from Lemma 3.2(iv) applied to the Coxeter group $W^{\prime}$ and the datum $\mathscr{C}^{\prime}=\left(\operatorname{span}\left(\Delta\left(W^{\prime}\right)\right), \Delta\left(W^{\prime}\right),(,)^{\prime}\right)$.

(ii) The desired result is trivially true if $x=y$, so we may assume that $x \neq y$. It is clear that $x \operatorname{dom}_{W} y$ implies that $x \operatorname{dom}_{W^{\prime}} y$. Conversely, suppose that $x \operatorname{dom}_{W^{\prime}} y$. Then (i) yields that $(x, y)=(x, y)^{\prime} \geq 1$. Thus Lemma 3.2(iv) yields that either $x \operatorname{dom}_{W} y$, or else $y \operatorname{dom}_{W} x$. If the latter is the case, then by the first part of the current proof, $y \operatorname{dom}_{W^{\prime}} x$, and hence it follows that $x=y$ (since dominance with respect to $W^{\prime}$ is a partial ordering), contradicting our choice of $x$ and $y$.

Next is a well-known result whose proof can be found in the remarks immediately before Lemma 2.3 of [Brink and Howlett 1993]:

Lemma 3.4. There is no nontrivial dominance between positive roots in the root system of a finite Coxeter group.

Next we have a technical result which is going to be used repeatedly in the rest of this paper.

Proposition 3.5. Let $\alpha, \beta \in \Phi^{+}$with $(\alpha, \beta) \leq-1$, and let $W^{\prime}$ be the dihedral reflection subgroup generated by $r_{\alpha}$ and $r_{\beta}$. Further, we set $\theta=\cosh ^{-1}(-(\alpha, \beta))$, and for each $i \in \mathbb{Z}$ adopt the notation

$$
c_{i}=\left\{\begin{array}{cc}
\frac{\sinh i \theta}{\sinh \theta} & \text { if } \theta \neq 0, \\
i & \text { if } \theta=0 .
\end{array}\right.
$$

(i) $W^{\prime}$ is infinite, and $\Phi\left(W^{\prime}\right)=\left\{c_{i \pm 1} \alpha+c_{i} \beta \mid i \in \mathbb{Z}\right\}$.

(ii) Suppose that $x, y \in \Phi\left(W^{\prime}\right)$. Then $(x, y) \in(-\infty,-1] \cup[1, \infty)$, and in particular, if $x \neq \pm y$ then $\left\langle\left\{r_{x}, r_{y}\right\}\right\rangle$ is an infinite dihedral reflection subgroup. More specifically,

(a) If $x=c_{n+1} \alpha+c_{n} \beta$ and $y=c_{m+1} \alpha+c_{m} \beta$, then

$$
(x, y)= \begin{cases}\cosh ((n-m) \theta) \geq 1 & \text { if } \theta \neq 0, \\ 1 & \text { if } \theta=0 .\end{cases}
$$

(b) If $x=c_{n+1} \alpha+c_{n} \beta$ and $y=c_{m-1} \alpha+c_{m} \beta$, then

$$
(x, y)= \begin{cases}-\cosh ((n+m) \theta) \leq-1 & \text { if } \theta \neq 0, \\ -1 & \text { if } \theta=0 .\end{cases}
$$


(c) If $x=c_{n-1} \alpha+c_{n} \beta$ and $y=c_{m+1} \alpha+c_{m} \beta$, then

$$
(x, y)= \begin{cases}-\cosh ((n+m) \theta) \leq-1 & \text { if } \theta \neq 0, \\ -1 & \text { if } \theta=0 .\end{cases}
$$

(d) If $x=c_{n-1} \alpha+c_{n} \beta$ and $y=c_{m-1} \alpha+c_{m} \beta$, then

$$
(x, y)= \begin{cases}\cosh ((n-m) \theta) \geq 1 & \text { if } \theta \neq 0, \\ 1 & \text { if } \theta=0 .\end{cases}
$$

(iii) If $x \in \Phi^{+}\left(W^{\prime}\right) \backslash\{\alpha, \beta\}$, then $D_{W^{\prime}}(x) \neq \varnothing$.

Proof. (i) Proposition 4.5.4(ii) of [Björner and Brenti 2005] implies that $W^{\prime}$ is infinite, and the rest of statement follows from direct calculations similar to those in Proposition 2.2.

(ii) This follows from (i) and a direct calculation.

(iii) If $x \in \Phi^{+}\left(W^{\prime}\right) \backslash\{\alpha, \beta\}$, part (i) yields that either $x=c_{n+1} \alpha+c_{n} \beta$ (for some $n \neq 0$ ), or else $x=c_{n-1} \alpha+c_{n} \beta$ (for some $n \neq 1$ ). Then part (ii) and Corollary 3.3(i) imply that we can find some $y \in \Phi^{+}\left(W^{\prime}\right) \backslash\{x\}$ such that $x \operatorname{dom}_{W^{\prime}} y$.

The other key object to be studied in this section is the numeric function $\infty$-height on $T$. As mentioned in the introduction, this function is defined in terms of infinite dihedral reflection subgroups of $W$, and in order to make a precise definition of this function we need a few technical results on infinite dihedral reflection subgroups. We begin with a well-known one, whose proof we include for completeness.

Proposition 3.6 [Dyer 1991]. Suppose that $\alpha, \beta \in \Phi^{+}$are distinct. Let

$$
W^{\prime}=\left\langle\left\{r_{\gamma} \mid \gamma \in(\mathbb{R} \alpha+\mathbb{R} \beta) \cap \Phi^{+}\right\}\right\rangle .
$$

Then $W^{\prime}$ is a dihedral reflection subgroup of $W$.

Proof. Suppose for a contradiction that $W^{\prime}$ is not dihedral. Then $\# S\left(W^{\prime}\right) \geq 3$, and let $x_{1}, x_{2}, x_{3} \in \Delta\left(W^{\prime}\right)$ be distinct. Theorem 2.9(ii) then yields that $\left(x_{i}, x_{j}\right) \leq 0$ whenever $i, j \in\{1,2,3\}$ are different. Clearly $x_{1}, x_{2}, x_{3}$ are all in the twodimensional subspace $\mathbb{R} \alpha+\mathbb{R} \beta$, and thus a contradiction would arise if we could show that $x_{1}, x_{2}, x_{3}$ are linearly independent. Let $c_{1}, c_{2}, c_{3} \in \mathbb{R}$ be such that $c_{1} x_{1}+c_{2} x_{2}+c_{3} x_{3}=0$. Since $x_{1}, x_{2}, x_{3} \in \Phi^{+}$, and $0 \notin \operatorname{PLC}(\Delta)$, it follows that $c_{1}, c_{2}, c_{3}$ cannot be all positive or all negative. Renaming $x_{1}, x_{2}, x_{3}$ if necessary, we have the following three possibilities:

$$
\begin{gathered}
c_{1}, c_{2} \geq 0 \text { and } c_{3}<0, \quad \text { or } \\
c_{1}, c_{2} \leq 0 \text { and } c_{3}>0, \quad \text { or } \\
c_{1}, c_{2}, c_{3}=0 .
\end{gathered}
$$


If (3-2) is the case then $0=\left(c_{1} x_{1}+c_{2} x_{2}+c_{3} x_{3}, x_{3}\right)<0$, and if (3-3) is the case then $0=\left(c_{1} x_{1}+c_{2} x_{2}+c_{3} x_{3}, x_{3}\right)>0$; both are clearly absurd. Hence (3-4) must be the case and $x_{1}, x_{2}, x_{3}$ are linearly independent, a contradiction, as required.

Let $\alpha, \beta \in \Phi^{+}$be distinct. Let $W^{\prime \prime}$ be an arbitrary dihedral reflection subgroup of $W$ containing the dihedral reflection subgroup $\left\langle\left\{r_{\alpha}, r_{\beta}\right\}\right\rangle$. Let $x, y$ be the canonical roots for $W^{\prime \prime}$. It can be readily checked that $\mathbb{R} x+\mathbb{R} y=\mathbb{R} \alpha+\mathbb{R} \beta$, and hence $x, y \in(\mathbb{R} \alpha+\mathbb{R} \beta) \cap \Phi^{+}$. It then follows that $W^{\prime \prime} \subseteq\left\langle\left\{r_{\gamma} \mid \gamma \in(\mathbb{R} \alpha+\mathbb{R} \beta) \cap \Phi^{+}\right\}\right\rangle$. This observation, together with Proposition 3.6, readily yields the following wellknown result:

Proposition 3.7. Every dihedral reflection subgroup $\left\langle\left\{r_{\alpha}, r_{\beta}\right\}\right\rangle$ of $W$ (where the elements $\alpha$ and $\beta$ of $\Phi^{+}$are distinct $)$, is contained in a unique maximal dihedral reflection subgroup, namely $\left\langle\left\{r_{\gamma} \mid \gamma \in \Phi^{+} \cap(\mathbb{R} \alpha+\mathbb{R} \beta)\right\}\right\rangle$.

Definition 3.8. (i) Define $\mu$ to be the set of all maximal dihedral reflection subgroups of $W$.

(ii) Define $\mathcal{M}_{\infty}$ to be the set $\left\{W^{\prime} \in \mathcal{M} \mid \# W^{\prime}=\infty\right\}$.

(iii) For each $t \in T$, define $M_{t}$ to be the set $\left\{W^{\prime} \in \mathcal{M} \mid t \in W^{\prime}\right\}$.

(iv) Let $W^{\prime}$ be a reflection subgroup of $W$, and let $t \in W^{\prime} \cap T$. Define the standard height, $h_{\left(W^{\prime}, S\left(W^{\prime}\right)\right.}(t)$, of $t$ with respect to the Coxeter system $\left(W^{\prime}, S\left(W^{\prime}\right)\right)$ to be

$$
\min \left\{\ell_{\left(W^{\prime}, S\left(W^{\prime}\right)\right)}(w) \mid w \in W^{\prime}, w \alpha_{t} \in \Delta\left(W^{\prime}\right)\right\} .
$$

For the standard height of $t$ with respect to the Coxeter system $(W, S)$, we simply write $h(t)$ in place of $h_{(W, S)}(t)$.

Remark 3.9. For arbitrary reflection subgroup $W^{\prime}$ of $W$, the depth function naturally applies to $\Phi^{+}\left(W^{\prime}\right)$ : if $x \in \Phi^{+}\left(W^{\prime}\right)$, then the depth of $x$ relative to $S\left(W^{\prime}\right)$ (written $\operatorname{dp}_{\left(W^{\prime}, S\left(W^{\prime}\right)\right)}(x)$ ) is defined to be

$$
\min \left\{\ell_{\left(W^{\prime}, S\left(W^{\prime}\right)\right)}(w) \mid w \in W^{\prime} \text { and } w x \in \Phi^{-}\left(W^{\prime}\right)\right\} .
$$

Now, for each $t \in W^{\prime} \cap T$, it is easily checked that

$$
\operatorname{dp}_{\left(W^{\prime}, S\left(W^{\prime}\right)\right)}\left(\alpha_{t}\right)=h_{\left(W^{\prime}, S\left(W^{\prime}\right)\right)}(t)+1,
$$

and hence applying Lemma 2.7(i) to the Coxeter system $\left(W^{\prime}, S\left(W^{\prime}\right)\right)$ yields that

$$
h_{\left(W^{\prime}, S\left(W^{\prime}\right)\right)}(t)=\frac{\ell_{\left(W^{\prime}, S\left(W^{\prime}\right)\right)}(t)-1}{2} .
$$

We include a proof of the next result for completeness:

Lemma 3.10 [Edgar 2009]. For each $t \in T$, we have

$$
T \backslash\{t\}=\biguplus_{W^{\prime} \in M_{t}}\left(\left(W^{\prime} \cap T\right) \backslash\{t\}\right) .
$$


Proof. It is readily checked that $T \backslash\{t\}=\bigcup_{W^{\prime} \in \mathcal{M}_{t}}\left(\left(W^{\prime} \cap T\right) \backslash\{t\}\right)$, and hence we only need to check that this union is indeed disjoint. Suppose for a contradiction that there are distinct $W_{1}, W_{2} \in \mathcal{M}_{t}$ with $r \in W_{1} \cap W_{2}$ for some $r \in T \backslash\{t\}$. Then clearly $\langle\{r, t\}\rangle \subseteq W_{1}$ and $\langle\{r, t\}\rangle \subseteq W_{2}$, contradicting Proposition 3.7.

From this and the canonical bijection $\psi: T \leftrightarrow \Phi^{+}$we immediately get:

Corollary 3.11. $\Phi^{+} \backslash\{\alpha\}=\biguplus_{W^{\prime} \in \mathcal{M}_{r_{\alpha}}}\left(\Phi^{+}\left(W^{\prime}\right) \backslash\{\alpha\}\right)$, for each $\alpha \in \Phi^{+}$.

Remark 3.12. In particular, the corollary implies that for $t \in T$, if $W_{1}, W_{2} \in \mathcal{M}_{t}$ are distinct, then $\Phi^{+}\left(W_{1}\right) \cap \Phi^{+}\left(W_{2}\right)=\left\{\alpha_{t}\right\}$.

Lemma 3.13 [Edgar 2009]. Let $t \in T$ be arbitrary. Then

$$
h(t)=\sum_{W^{\prime} \in M_{t}} h_{\left(W^{\prime}, S\left(W^{\prime}\right)\right)}(t) .
$$

Proof. For any reflection $t \in T$, Corollary 3.11 yields that

$$
\left\{\alpha \in \Phi^{+} \mid t \alpha \in \Phi^{-}\right\}=\left\{\alpha_{t}\right\} \cup\left(\biguplus_{W^{\prime} \in \mathcal{M}_{t}}\left\{\alpha \in \Phi^{+}\left(W^{\prime}\right) \backslash\left\{\alpha_{t}\right\} \mid t \alpha \in \Phi^{-}\right\}\right)
$$

Since $h(t)=\frac{1}{2}(\ell(t)-1)=\frac{1}{2}(\# N(t)-1)$, it follows from $(3-6)$ that

$$
\begin{array}{rlr}
h(t) & =\frac{1}{2}\left(\sum_{W^{\prime} \in \mathcal{M}_{t}} \#\left\{\alpha \in \Phi^{+}\left(W^{\prime}\right) \backslash\left\{\alpha_{t}\right\} \mid t \alpha \in \Phi^{-}\left(W^{\prime}\right)\right\}\right) \\
& =\sum_{W^{\prime} \in \mathcal{M}_{t}} \frac{1}{2}\left(\ell_{\left(W^{\prime}, S\left(W^{\prime}\right)\right)}(t)-1\right) & (\text { by }(2-4)) \\
& =\sum_{W^{\prime} \in \mathcal{M}_{t}} h_{\left(W^{\prime}, S\left(W^{\prime}\right)\right)}(t) & \text { (by }(3-5)) .
\end{array}
$$

Definition 3.14 [Edgar 2009]. For $t \in T$, define the $\infty$-height of $t$ to be

$$
h^{\infty}(t)=\sum_{W^{\prime} \in M_{t} \cap M_{\infty}} h_{\left(W^{\prime}, S\left(W^{\prime}\right)\right)}(t),
$$

and for each nonnegative integer $n$, we define

$$
T_{n}=\left\{t \in T \mid h^{\infty}(t)=n\right\} .
$$

From this definition it is not clear whether, for a specific nonnegative integer $n$, there is a reflection $t \in T$ with $h^{\infty}(t)=n$. It turns out that a number of basic questions like this can be solved with the aid of the results obtained in [Fu 2012] once we prove the following:

Theorem 3.15. For each nonnegative integer $n$, there is a bijection $T_{n} \leftrightarrow D_{n}$ given by $t \leftrightarrow \alpha_{t}$. 
The proof of the theorem will be deferred until we have all the necessary tools. Proposition 3.16. Suppose that $t \in T$, and let $W^{\prime}$ be an infinite dihedral reflection subgroup containing $t$. If $h_{\left(W^{\prime}, S\left(W^{\prime}\right)\right)}(t) \geq 1$, then there exists some $x \in \Phi^{+}\left(W^{\prime}\right)$ distinct from $\alpha_{t}$ and satisfying $\alpha_{t} \operatorname{dom}_{W} x$.

Proof. Observe that the condition $h_{\left(W^{\prime}, S\left(W^{\prime}\right)\right)}(t) \geq 1$ is equivalent to $\alpha_{t} \notin \Delta\left(W^{\prime}\right)$, and hence the required result follows immediately from Proposition 3.5(iii).

The following proposition will be a key step to prove Theorem 3.15:

Proposition 3.17. Let $W^{\prime}$ be an infinite dihedral reflection subgroup, and let $\Delta\left(W^{\prime}\right)=\{\alpha, \beta\}$.

(i) There are two disjoint dominance chains in $\Phi\left(W^{\prime}\right)$, namely:

$$
\begin{aligned}
& \cdots \operatorname{dom}_{W} r_{\alpha} r_{\beta} r_{\alpha}(\beta) \operatorname{dom}_{W} r_{\alpha} r_{\beta}(\alpha) \operatorname{dom}_{W} r_{\alpha}(\beta) \operatorname{dom}_{W} \alpha \\
& \operatorname{dom}_{W}(-\beta) \operatorname{dom}_{W} r_{\beta}(-\alpha) \operatorname{dom}_{W} r_{\beta} r_{\alpha}(-\beta) \operatorname{dom}_{W} \cdots
\end{aligned}
$$

and

$$
\begin{aligned}
\cdots \operatorname{dom}_{W} r_{\beta} r_{\alpha} r_{\beta}(\alpha) \operatorname{dom}_{W} r_{\beta} r_{\alpha}(\beta) \operatorname{dom}_{W} r_{\beta}(\alpha) \operatorname{dom}_{W} \beta \\
\\
\quad \operatorname{dom}_{W}(-\alpha) \operatorname{dom}_{W} r_{\alpha}(-\beta) \operatorname{dom}_{W} r_{\alpha} r_{\beta}(-\alpha) \operatorname{dom}_{W} \cdots .
\end{aligned}
$$

In particular, each root in $\Phi\left(W^{\prime}\right)$ lies in exactly one of these two chains, and the negative of any element of one chain lies in the other. The roots in $\Phi\left(W^{\prime}\right)$ dominated by either $\alpha$ or $\beta$ are all negative.

(ii) If $x \in \Phi\left(W^{\prime}\right)$ then $\# D_{W^{\prime}}(x)=h_{\left(W^{\prime}, S\left(W^{\prime}\right)\right)}\left(r_{x}\right)$.

Proof. (i) Theorem 2.9(ii) and [Björner and Brenti 2005, Proposition 4.5.4 (ii)] yield that $(\alpha, \beta) \leq-1$. Hence it follows from Lemma 3.2(iv) that $\alpha \operatorname{dom}_{W}-\beta$ and $\beta$ dom $_{W}-\alpha$. Then we can immediately verify the existence of the two dominance chains (3-7) and (3-8), and from these two chains the remaining statements in (i) follow readily.

(ii) The required result follows immediately from the definition of $h_{\left(W^{\prime}, S\left(W^{\prime}\right)\right)}\left(r_{x}\right)$ and the two dominance chains (3-7) and (3-8).

Proposition 3.18. Suppose that $x, y \in \Phi^{+}$are distinct with $x \operatorname{dom}_{W} y$, and let $W^{\prime}$ be a dihedral reflection subgroup containing $r_{x}$ and $r_{y}$. Then $h_{\left(W^{\prime}, S\left(W^{\prime}\right)\right)}\left(r_{x}\right) \geq 1$. Proof. It follows from Corollary 3.3(ii) that $x \operatorname{dom}_{W^{\prime}} y$, so Lemma 3.4 above yields that $W^{\prime}$ is an infinite dihedral reflection subgroup. Let $\{\alpha, \beta\}=\Delta\left(W^{\prime}\right)$. We know from Proposition 3.17(i) that the roots in $\Phi\left(W^{\prime}\right)$ dominated by either $\alpha$ or $\beta$ are all negative, and since $x \operatorname{dom}_{W} y \in \Phi^{+}$, it follows that $x \notin\{\alpha, \beta\}$. Hence by definition $h_{\left(W^{\prime}, S\left(W^{\prime}\right)\right)}\left(r_{x}\right) \geq 1$. 
From the last two propositions we may deduce the following special case of Theorem 3.15:

Lemma 3.19. There is a bijection $T_{0} \leftrightarrow D_{0}$ given by $t \leftrightarrow \alpha_{t}$.

Proof. Let $t \in T_{0}$, and suppose for a contradiction that $\alpha_{t} \notin D_{0}$. Then there exists $s \in T \backslash\{t\}$ such that $\alpha_{t}$ dom $_{W} \alpha_{s}$. Let $W^{\prime}$ be the unique maximal dihedral reflection subgroup of $W$ containing $\langle\{s, t\}\rangle$. Proposition 3.18 yields that $h_{\left(W^{\prime}, S\left(W^{\prime}\right)\right)}(t) \geq 1$. Since $\alpha_{t}$ dom $_{W^{\prime}} \alpha_{s}$, it follows from Lemma 3.4 that $W^{\prime} \in M_{\infty}$, and consequently $h^{\infty}(t) \geq 1$, contradicting the assumption that $t \in T_{0}$.

Conversely, suppose that $\alpha_{t} \in D_{0}$, and suppose for a contradiction that $t \notin T_{0}$. Then there exists some $W^{\prime} \in M_{t} \cap M_{\infty}$ with $h_{\left(W^{\prime}, S\left(W^{\prime}\right)\right)}(t) \geq 1$. But then Proposition 3.16 yields that $\alpha_{t} \notin D_{0}$, producing a contradiction as required.

Observe that Proposition 3.17(ii) can be equivalently stated as:

Proposition 3.20. Suppose that $t \in T$, and suppose that $W^{\prime}$ is an infinite dihedral reflection subgroup containing $t$. Then

$$
\# D_{W^{\prime}}\left(\alpha_{t}\right)=h_{\left(W^{\prime}, S\left(W^{\prime}\right)\right)}(t) .
$$

Proposition 3.21. Suppose that $t \in T$ is arbitrary. Then

$$
\biguplus_{W^{\prime} \in M_{t} \cap M_{\infty}} D_{W^{\prime}}\left(\alpha_{t}\right)=D\left(\alpha_{t}\right) .
$$

Proof. First we observe that Remark 3.12 yields that the union of the sets $D_{W^{\prime}}\left(\alpha_{t}\right)$ over all $W^{\prime}$ in $\mu_{t} \cap \mu_{\infty}$ is indeed disjoint.

It is clear that $\biguplus_{W^{\prime} \in M_{t} \cap M_{\infty}} D_{W^{\prime}}\left(\alpha_{t}\right) \subseteq D\left(\alpha_{t}\right)$.

Conversely, suppose that $x \in D\left(\alpha_{t}\right)$. Let $W^{\prime}$ be the unique maximal dihedral reflection subgroup of $W$ containing $\left\langle\left\{t, r_{x}\right\}\right\rangle$. Then Corollary 3.3(ii) yields that $\alpha_{t} \operatorname{dom}_{W^{\prime}} x$. Finally since there is no nontrivial dominance in any finite Coxeter group, it follows that $W^{\prime} \in \mathcal{M}_{\infty}$, as required.

Now we prove that for any reflection $t \in W$, its $\infty$-height $h^{\infty}(t)$ equals the number of positive roots strictly dominated by $\alpha_{t}$ :

Theorem 3.22. Let $t \in T$ be arbitrary. Then $h^{\infty}(t)=\# D\left(\alpha_{t}\right)$.

Proof. It follows from Proposition 3.20 and Proposition 3.21 that

$$
h^{\infty}(t)=\sum_{W^{\prime} \in M_{t} \cap M_{\infty}} h_{\left(W^{\prime}, S\left(W^{\prime}\right)\right)}(t)=\sum_{W^{\prime} \in M_{t} \cap M_{\infty}} \# D_{W^{\prime}}\left(\alpha_{t}\right)=\# D\left(\alpha_{t}\right) .
$$

Proof of Theorem 3.15. The theorem follows immediately from Theorem 3.22.

Combining [Fu 2012, Theorem 3.8, Corollary 3.9, and Corollary 3.21] with Theorem 3.15 we may deduce: 
Corollary 3.23. (i) For each positive integer $n$,

$$
T_{n} \subseteq\left\{t t^{\prime} t \mid t \in T_{0} \text { and } t^{\prime} \in T_{m} \text { for some } m \leq n-1\right\} .
$$

(ii) Suppose that $W$ is an infinite Coxeter group with $\# S<\infty$. Then

$$
0<\# T_{n} \leq\left(\# T_{0}\right)^{n+1}-\left(\# T_{0}\right)^{n}
$$

for each positive integer $n$.

Remark 3.24. An upper bound for $\# T_{0}\left(=\# D_{0}\right)$ is given in [Brink and Howlett 1993]; furthermore, for any fixed finitely generated Coxeter group, this number can be explicitly calculated following the methods presented in [Brink 1998].

\section{Dominance and imaginary cone}

Kac introduced the concept of an imaginary cone in the study of the imaginary roots of Kac-Moody Lie algebras. In [Kac 1990, Chapter 5] the imaginary cone of a Kac-Moody Lie algebra was defined to be the positive cone on the positive imaginary roots. The generalization of imaginary cones to arbitrary Coxeter groups was first introduced in [Hée 1990], and subsequently reproduced in [Hée 1993]. This generalization has also been studied in [Dyer 2012] and [Edgar 2009]. In this section we investigate the connections between this generalized imaginary cone and dominance in Coxeter groups; in particular, we show that whenever $x$ and $y$ are roots of a Coxeter group, then $x \operatorname{dom}_{W} y$ if and only if $x-y$ lies in the imaginary cone of that Coxeter group.

Let $(W, S)$ be the abstract Coxeter system associated to the Coxeter datum $\mathscr{C}=(V, \Delta,()$,$) , and let \Phi$ be the corresponding root system. Let $X$ be a vector subspace of $V$. In this paper, a cone is assumed to be a convex cone. For any cone $C$ in $X$, we define $C^{*}=\{f \in \operatorname{Hom}(X, \mathbb{R}) \mid f(v) \geq 0$ for all $v \in C\}$ and call $C^{*}$ the dual of $C$; and for any cone $F \in \operatorname{Hom}(X, \mathbb{R})$, we define $F^{*}=\{v \in X \mid f(v) \geq$ 0 for all $f \in F\}$ and call $F^{*}$ the dual of $F$. If $W$ acts on $X$, then $\operatorname{Hom}(X, \mathbb{R})$ bears the contragredient representation of $W$ in the following way: if $w \in W$ and $f \in \operatorname{Hom}(X, \mathbb{R})$ then $w f \in \operatorname{Hom}(X, \mathbb{R})$ is given by the rule $(w f)(v)=f\left(w^{-1} v\right)$ for all $v \in X$. It is readily checked that for a cone $C$ in $X$, we have $C \subseteq C^{* *}$, and also for any $w \in W$, we have $(w C)^{*}=w C^{*}$.

The following is a well-known result whose proof can be found in [Howlett 1996, Lecture 1, Note (c)]:

Lemma 4.1. Suppose that $X$ is a real vector space of finite dimension, and let $C$ be a cone in $X$. Then $\left(C^{*}\right)^{*}=\bar{C}$, where $\bar{C}$ is the topological closure of $C$ in $X$ (with respect to the standard topology on $X$ ).

Set $P=\operatorname{PLC}(\Delta) \cup\{0\}$. It is clear that $P$ is a cone in $V$. We define the Tits cone of $W$ in the same way as in Section 5.13 of [Humphreys 1990]: 
Definition 4.2. The Tits cone of the Coxeter group $W$ is the $W$-invariant set

$$
U=\bigcup_{w \in W} w P^{*}
$$

It is not obvious from this definition that the Tits cone is indeed a cone; however, this is made clear by the following result:

\section{Proposition 4.3.}

(4-1) $U=\left\{f \in \operatorname{Hom}(\operatorname{span}(\Delta), \mathbb{R}) \mid f(x) \geq 0\right.$ for all but finitely many $\left.x \in \Phi^{+}\right\}$.

Proof. Denote the set on the right-hand side of (4-1) by $Y$, and for each $f \in$ $\operatorname{Hom}(\operatorname{span}(\Delta), \mathbb{R})$ define $\operatorname{Neg}(f)$ by $\operatorname{Neg}(f)=\left\{x \in \Phi^{+} \mid f(x)<0\right\}$.

If $f \in U$ then $f=w g$ for some $w \in W$ and $g \in P^{*}$, and it is readily checked that $\operatorname{Neg}(f) \subseteq N\left(w^{-1}\right)$. Since $N\left(w^{-1}\right)$ is a finite set, it follows that $f \in Y$, and hence $U \subseteq Y$. Conversely, suppose that $f \in Y$. If $\operatorname{Neg}(f)=\varnothing$ then $f \in P^{*} \subseteq U$. Thus we may assume that \# $\operatorname{Neg}(f)>0$, and proceed by induction. Observe that then there exists some $\alpha \in \Delta$ such that $f(\alpha)<0$. It is then readily checked that \# $\operatorname{Neg}\left(r_{\alpha} f\right)=\# \operatorname{Neg}(f)-1$, and hence it follows from the inductive hypothesis that $r_{\alpha} f \in U$. Since $U$ is $W$-invariant, it follows that $f \in U$, and hence $Y \subseteq U$.

Lemma 4.4. $U^{*}=\bigcap_{w \in W} w\left(P^{*}\right)^{*}$. Furthermore, $U^{*}=\bigcap_{w \in W} w P$ whenever $\Delta$ is a finite set.

Proof. Write $U^{*}=\{v \in V \mid f(v) \geq 0$, for all $f \in U\}$

$$
\begin{aligned}
& =\left\{v \in V \mid(w \phi)(v) \geq 0 \text { for all } \phi \in P^{*} \text { and all } w \in W\right\} \\
& =\left\{v \in V \mid \phi\left(w^{-1} v\right) \geq 0, \text { for all } \phi \in P^{*} \text { and all } w \in W\right\} \\
& =\bigcap_{w \in W}\left\{v \in V \mid \phi\left(w^{-1} v\right) \geq 0 \text { for all } \phi \in P^{*}\right\} \\
& =\bigcap_{w \in W}\left\{w v \in V \mid \phi(v) \geq 0 \text { for all } \phi \in P^{*}\right\} .
\end{aligned}
$$

Thus

$$
U^{*}=\bigcap_{w \in W}\left\{w v \in V \mid v \in\left(P^{*}\right)^{*}\right\} .
$$

Let $X=\operatorname{span}(\Delta)$. If $\# \Delta$ is finite then it follows from Lemma 4.1 that $\left(P^{*}\right)^{*}=\bar{P}$. It is clear that $P$ is topologically closed; hence (4-2) yields that $U^{*}=\bigcap_{w \in W} w P$ when $\Delta$ is a finite set.

Lemma 4.5. Suppose that $v \in V$ has the property that $(a, v) \leq 0$ for all $a \in \Delta$. Then $w v-v \in P$ for all $w \in W$. Moreover, if $v \in P$ then $v \in U^{*}$.

Proof. Use induction on $\ell(w)$. If $\ell(w)=0$ then there is nothing to prove. If $\ell(w) \geq 1$ then we may write $w=w^{\prime} r_{a}$ where $w^{\prime} \in W$ and $a \in \Delta$ with $\ell(w)=\ell\left(w^{\prime}\right)+1$. 
Then Proposition 2.3 yields that $w^{\prime} a \in \Phi^{+} \subseteq P$, and we have

$$
w v-v=\left(w^{\prime} r_{a}\right) v-v=w^{\prime}(v-2(v, a) a)-v=\left(w^{\prime} v-v\right)-2(a, v) w^{\prime} a .
$$

By the inductive hypothesis, $w^{\prime} v-v \in P$. Since $(a, v) \leq 0$, it follows from the above that $w v-v \in P$.

If $v \in P$ then $w v=(w v-v)+v \in P$ for all $w \in W$, and hence

$$
v \in \bigcap_{w \in W} w^{-1} P \subseteq U^{*} .
$$

Proposition 4.6 [Fu 2012, Proposition 3.4]. Suppose that $x, y \in \Phi$ are distinct with $x \operatorname{dom}_{W} y$. Let $W^{\prime}$ be the dihedral reflection subgroup generated by $r_{x}$ and $r_{y}$, and let $\Delta\left(W^{\prime}\right)=\{\alpha, \beta\}$. Then there exists some $w \in W^{\prime}$ such that

$$
\left\{\begin{array} { l } 
{ w x = \alpha , } \\
{ w y = - \beta , }
\end{array} \quad \text { or } \quad \left\{\begin{array}{l}
w x=\beta, \\
w y=-\alpha .
\end{array}\right.\right.
$$

In particular, $(x, y)=-(a, b)$.

Proposition 4.7. Suppose that $x, y \in \Phi$ such that $x \operatorname{dom}_{W} y$. Then $w(x-y) \in$ $\operatorname{PLC}(\Delta)$ for all $w \in W$, that is, $x-y \in U^{*}$.

Proof. The assertion is trivially true if $x=y$, so we may assume that $x \neq y$. Since $x \operatorname{dom}_{W} y$, Lemma 3.2(iv) yields that $(x, y) \geq 1$. Let $W^{\prime}$ be the (infinite) dihedral subgroup of $W$ generated by $r_{x}$ and $r_{y}$. Let $S\left(W^{\prime}\right)=\{s, t\}$ and $\triangle\left(W^{\prime}\right)=\left\{\alpha_{s}, \alpha_{t}\right\}$. Proposition 4.6 yields that $\left(\alpha_{s}, \alpha_{t}\right)=-(x, y) \leq-1$. Set $c_{i}$ as in Proposition 3.5 for each $i \in \mathbb{Z}$. Since $x \operatorname{dom}_{W} y$, it follows that $(x, y) \geq 1$, and Proposition 3.5(ii) then yields that there exist integers $m$ and $n$ such that

$$
\left\{\begin{array} { l } 
{ x = c _ { n + 1 } \alpha _ { s } + c _ { n } \alpha _ { t } , } \\
{ y = c _ { m + 1 } \alpha _ { s } + c _ { m } \alpha _ { t } , }
\end{array} \quad \text { or } \quad \left\{\begin{array}{l}
x=c_{n-1} \alpha_{s}+c_{n} \alpha_{t}, \\
y=c_{m-1} \alpha_{s}+c_{m} \alpha_{t} .
\end{array}\right.\right.
$$

Next we shall show that $n>m$. Suppose for a contradiction that $m \geq n$. Then either $x=y($ when $n=m)$ or else there will be a $w \in W^{\prime}$ such that $w x \in \Phi\left(W^{\prime}\right) \cap \Phi^{-}$and yet $w y \in \Phi\left(W^{\prime}\right) \cap \Phi^{+}$(when $n<m$ ), both contradicting the fact that $x \operatorname{dom}_{W} y$. Since $c_{n}>c_{m}$ whenever $n>m$, it follows that $x-y \in \operatorname{PLC}(\Delta)$. Given the $W$ invariance of dominance, for any $w \in W$, repeat the argument with $x$ replaced by $w x$ and $y$ replaced by $w y$, we may conclude that $w(x-y) \in \operatorname{PLC}(\Delta) \subseteq\left(P^{*}\right)^{*}$. It then follows from Lemma 4.4 that $x-y \in U^{*}$.

When $\# \Delta$ is finite, it can be checked that Lemma 4.4 yields that whenever $x$, $y \in \Phi$ such that $x-y \in U^{*}$, then $x \operatorname{dom}_{W} y$. In fact we can remove this finiteness condition and still prove the same result, and to do so we need some special notations and a few extra elementary results. We thank the referee of this paper for prompting us to look in this direction. 
Notations 4.8. For a subset $I$ of $S$ we set $\Delta_{I}=\left\{x \in \Delta \mid r_{x} \in I\right\} ; V_{I}=\operatorname{span}\left(\Delta_{I}\right)$; $W_{I}=\langle I\rangle$; and $P_{I}=\operatorname{PLC}\left(\Delta_{I}\right) \cup\{0\}$. Furthermore, we set

$$
\begin{aligned}
P_{I}^{*} & =\left\{f \in \operatorname{Hom}\left(V_{I}, \mathbb{R}\right) \mid f(x) \geq 0 \text { for all } x \in P_{I}\right\} ; \\
P_{I}^{* *} & =\left\{x \in V_{I} \mid f(x) \geq 0 \text { for all } f \in P_{I}^{*}\right\} .
\end{aligned}
$$

Then $\mathscr{C}_{I}=\left(V_{I}, \Delta_{I},(,)_{I}\right)$ (where $(,)_{I}$ is the restriction of $($,$\left.) on V_{I}\right)$ is a Coxeter datum with corresponding Coxeter system $\left(W_{I}, I\right)$, and we call $W_{I}$ the standard parabolic subgroup of $W$ corresponding to $I$. Clearly $W_{I}$ preserves $V_{I}$.

Lemma 4.9. Suppose that $I$ is a subset of $S$. Then $P^{* *} \cap V_{I} \subseteq P_{I}^{* *}$.

Proof. Write $V=V_{I} \oplus V_{I}^{\prime}$, where $V_{I}^{\prime}$ is a vector space complement of $V_{I}$. Then every $v \in V$ is uniquely written as $v=v_{I}+v_{I}^{\prime}$, where $v_{I} \in V_{I}$ and $v_{I}^{\prime} \in V_{I}^{\prime}$. Every $g \in P_{I}^{*}$ gives rise to a $g^{\prime} \in P^{*}$ as follows: for any $v \in V$, simply set $g^{\prime}(v)=g\left(v_{I}\right)$. Now let $x \in P^{* *} \cap V_{I}$ and $f \in P_{I}^{*}$ be arbitrary. Then $f(x)=f^{\prime}(x) \geq 0$, since $f^{\prime} \in P^{*}$ and $x \in P^{* *}$. Hence $x \in P_{I}^{* *}$, and so $P^{* *} \cap V_{I} \subseteq P_{I}^{* *}$.

Proposition 4.10. Let $x, y \in \Phi$. Then $x-y \in U^{*}$ if and only if $x \operatorname{dom}_{W} y$.

Proof. By Proposition 4.7 we only need to prove that when $x$ and $y$ are both roots then $x-y \in U^{*}$ implies that $x \operatorname{dom}_{W} y$. The assertion certainly holds if $x=y$, thus we only need to check the case when $x \neq y$.

Since dominance and $U^{*}$ are both $W$-invariant, it follows that we only need to prove the following statement: if $x \in \Phi^{-}$then $y \in \Phi^{-}$too.

Take $I=\left\{r_{\alpha} \mid \alpha \in \operatorname{supp}(x) \cup \operatorname{supp}(y)\right\}$, and note that in particular, $I$ is a finite set. Now in view of Lemma 4.4, Lemma 4.9 and the fact that $W_{I}$ preserves $V_{I}$ we have

$$
\begin{aligned}
x-y \in\left(\bigcap_{w \in W} w P^{* *}\right) \cap V_{I} & \subseteq\left(\bigcap_{w \in W_{I}} w P^{* *}\right) \cap V_{I} \subseteq \bigcap_{w \in W_{I}} w\left(P^{* *} \cap V_{I}\right) \\
& \subseteq \bigcap_{w \in W_{I}} w P_{I}^{* *}=\bigcap_{w \in W_{I}} w P_{I},
\end{aligned}
$$

where the equality follows from Lemma 4.1 , since $I$ is a finite set. Thus $x-y \in P_{I}$, and this implies, precisely, that $y \in \Phi^{-}$whenever $x \in \Phi^{-}$.

Next we have a technical result which is a key component of the main theorem of this section.

Proposition 4.11. Suppose that $x, y \in \Phi$ are distinct with $x \operatorname{dom}_{W} y$. Then there exists some $w \in W$ such that $w x \in \Phi^{+}, w y \in \Phi^{-}$and $(w(x-y), z) \leq 0$ for all $z \in \Phi^{+}$.

Proof. Clearly it is enough to show that under such assumptions there exists some $w \in W$ with $w x \in \Phi^{+}, w y \in \Phi^{-}$and $(w(x-y), z) \leq 0$ for all $z \in \Delta$. 
Let $W^{\prime}$ be the (infinite) dihedral reflection subgroup of $W$ generated by $r_{x}$ and $r_{y}$, and let $\triangle\left(W^{\prime}\right)=\left\{a_{0}, b_{0}\right\}$. Clearly $a_{0}, b_{0} \in \Phi^{+}$, and Proposition 4.6 yields that $\left(a_{0}, b_{0}\right)=-(x, y) \leq-1$; furthermore, there is some $u \in\left\langle\left\{r_{x}, r_{y}\right\}\right\rangle$ such that

$$
\left\{\begin{array} { l } 
{ u ( x ) = a _ { 0 } , } \\
{ u ( y ) = - b _ { 0 } , }
\end{array} \quad \text { or } \quad \left\{\begin{array}{l}
u(x)=b_{0}, \\
u(y)=-a_{0} .
\end{array}\right.\right.
$$

At any rate, $u(x-y)=a_{0}+b_{0}$. Since the $W$-action preserves (, ), it follows that $\left(a_{0}, a_{0}\right)=1=\left(b_{0}, b_{0}\right)$, and hence $\left(a_{0}+b_{0}, a_{0}\right) \leq 0$ and $\left(a_{0}+b_{0}, b_{0}\right) \leq 0$. However there may exist some $c_{1} \in \Delta$ with $\left(a_{0}+b_{0}, c_{1}\right)>0$. If this is the case, set $a_{1}=r_{c_{1}} a_{0}$ and $b_{1}=r_{c_{1}} b_{0}$. Recall that $\left(d, c_{1}\right) \leq 0$ for all $d \in \Delta \backslash\left\{c_{1}\right\}$, so it follows that

$$
c_{1} \in \operatorname{supp}\left(a_{0}\right) \cup \operatorname{supp}\left(b_{0}\right) .
$$

Since $\left(a_{0}+b_{0}, c_{1}\right)>0$, whereas $\left(a_{0}+b_{0}, a_{0}\right) \leq 0$ and $\left(a_{0}+b_{0}, b_{0}\right) \leq 0$, it follows that $a_{0} \neq c_{1}$ and $b_{0} \neq c_{1}$. Therefore we see that $a_{1}, b_{1} \in \Phi^{+}$, and $\left(a_{1}, b_{1}\right)=\left(a_{0}, b_{0}\right) \leq-1$. Consequently Theorem 2.9(ii) yields that $a_{1}, b_{1}$ are the canonical roots for the root subsystem $\Phi\left(\left\langle\left\{r_{a_{1}}, r_{b_{1}}\right\}\right\rangle\right)$. Since $r_{c_{1}}\left(a_{0}+b_{0}\right)=$ $a_{0}+b_{0}-2\left(a_{0}+b_{0}, c_{1}\right) c_{1}$ and $\left(a_{0}+b_{0}, c_{1}\right)>0$, it follows that

$$
\operatorname{supp}\left(a_{1}\right) \cup \operatorname{supp}\left(b_{1}\right) \subseteq \operatorname{supp}\left(a_{0}\right) \cup \operatorname{supp}\left(b_{0}\right)
$$

and

$$
\sum_{a \in \Delta} \operatorname{coeff}_{a}\left(a_{1}\right)+\sum_{a \in \Delta} \operatorname{coeff}_{a}\left(b_{1}\right)<\sum_{a \in \Delta} \operatorname{coeff}_{a}\left(a_{0}\right)+\sum_{a \in \Delta} \operatorname{coeff}_{a}\left(b_{0}\right) .
$$

Moreover, since $\left(a_{0}+b_{0}, c_{1}\right)>0$, it follows that at least one of $\left(a_{0}, c_{1}\right)$ or $\left(b_{0}, c_{1}\right)$ must be strictly positive. Hence Lemma 2.7 yields that

$$
d p\left(a_{1}\right)+d p\left(b_{1}\right) \leq d p\left(a_{0}\right)+d p\left(b_{0}\right) .
$$

Repeating this process, we can obtain new pairs of positive roots $\left\{a_{2}, b_{2}\right\}, \ldots$, $\left\{a_{m-1}, b_{m-1}\right\},\left\{a_{m}, b_{m}\right\}$ with

$\operatorname{supp}\left(a_{m}\right) \cup \operatorname{supp}\left(b_{m}\right) \subseteq \operatorname{supp}\left(a_{m-1}\right) \cup \operatorname{supp}\left(b_{m-1}\right) \subseteq \cdots \subseteq \operatorname{supp}\left(a_{0}\right) \cup \operatorname{supp}\left(b_{0}\right)$

and $\operatorname{dp}\left(a_{m}\right)+\operatorname{dp}\left(b_{m}\right) \leq \mathrm{dp}\left(a_{m-1}\right)+\operatorname{dp}\left(b_{m-1}\right) \leq \cdots \leq \mathrm{dp}\left(a_{0}\right)+\mathrm{dp}\left(b_{0}\right)$, so long as we can find a $c_{m} \in \Delta$ such that $\left(a_{m-1}+b_{m-1}, c_{m}\right)>0$. This process only terminates at a pair $\left\{a_{n}, b_{n}\right\}$ for some $n$ if $\left(a_{n}+b_{n}, z\right) \leq 0$ for all $z \in \Delta$. Now if we could show that this process terminates at some such $\left\{a_{n}, b_{n}\right\}$ after a finite number of iterations, then we have in fact found a $w \in W$ given by

$$
w=r_{c_{n}} r_{c_{n-1}} \cdots r_{c_{1}} u, \quad \text { where } u \text { is as in (4-3), }
$$


satisfying

$$
(w(x-y), z)=\left(r_{c_{n}} \cdots r_{c_{1}}\left(a_{0}+b_{0}\right), z\right)=\left(a_{n}+b_{n}, z\right) \leq 0
$$

for all $z \in \Delta$.

Observe that the set of positive roots having depth less than or equal to the specific bound $\operatorname{dp}\left(a_{0}\right)+\operatorname{dp}\left(b_{0}\right)$ and support in the fixed finite subset $\operatorname{supp}\left(a_{0}\right) \cup \operatorname{supp}\left(b_{0}\right)$ of $\Delta$ is finite; indeed, Lemma 2.7(ii) implies that there are at most

$$
\#\left(\operatorname{supp}\left(a_{0}\right) \cup \operatorname{supp}\left(b_{0}\right)\right)^{\mathrm{dp}\left(a_{0}\right)+\mathrm{dp}\left(b_{0}\right)}
$$

such positive roots. Hence it follows that the possible pairs of positive roots $\left\{a_{i}, b_{i}\right\}$ obtainable in the process above must be finite too. Since

$$
\sum_{a \in \Delta} \operatorname{coeff}_{a}\left(a_{j}\right)+\sum_{a \in \Delta} \operatorname{coeff}_{a}\left(b_{j}\right)<\sum_{a \in \Delta} \operatorname{coeff}_{a}\left(a_{i}\right)+\sum_{a \in \Delta} \operatorname{coeff}_{a}\left(b_{i}\right)
$$

for all $j>i$, it follows that the sequence $\left\{a_{0}, b_{0}\right\},\left\{a_{1}, b_{1}\right\}, \ldots$ must terminate at $\left\{a_{n}, b_{n}\right\}$ for some finite $n$, as required.

Finally, keeping $w$ as in (4-5), we see from the construction above that either $w x=a_{n} \in \Phi^{+}$and $w y=-b_{n} \in \Phi^{-}$, or $w x=b_{n} \in \Phi^{+}$and $w y=-a_{n} \in \Phi^{-}$.

Definition 4.12. We define the imaginary cone $Q$ of $W$ by

$$
Q=\left\{v \in U^{*} \mid(v, a) \leq 0 \text { for all but finitely many } a \in \Phi^{+}\right\} .
$$

The following result was obtained independently by Dyer as a consequence of [Dyer 2012, Theorem 6.3], stating that the imaginary cone of a reflection subgroup is contained in that of the overgroup.

Theorem 4.13. Suppose that $x, y \in \Phi$ such that $x \operatorname{dom}_{W} y$. Then $x-y \in Q$.

Proof. By Proposition 4.7 we know that $x-y \in U^{*}$, thus to prove the desired result, we only need to show that $(x-y, z) \leq 0$ for all but finitely many $z \in \Phi^{+}$. Suppose that $z \in \Phi^{+}$such that $(x-y, z)>0$. Let $w \in W$ be as in Proposition 4.11. Then $(w(x-y), w z)>0$, and by Proposition 4.11 this is possible only if $z \in N(w)$. Since $\# N(w)$ is clearly finite (equal to $\ell(w)$ ), it follows that indeed $(x-y, z) \leq 0$ for all but finitely many $z \in \Phi^{+}$.

Remark 4.14. The above theorem is a special case of Dyer's result when the subgroup is dihedral. In fact, Dyer's result, when applied to dihedral reflection subgroups, implies that if $x$ and $y$ are roots with $x \operatorname{dom}_{W} y$, then $x-c y \in Q$ for an explicit range of $c \in \mathbb{R}$ depending on the value of $(x, y)$. Our formulation was first suggested to us by Howlett and Dyer, and we gratefully acknowledge their help.

Theorem 4.13, combined with Proposition 4.10, immediately implies this:

Corollary 4.15. Let $x, y \in \Phi$. Then $x-y \in Q$ if and only if $x \operatorname{dom}_{W} y$. 
Remark 4.16. By Proposition 4.10 and Corollary 4.15, when $x, y \in \Phi$, it is impossible for $x-y$ to be in $U^{*} \backslash Q$.

Corollary 4.17. Suppose that $x, y \in \Phi$ are distinct. The following are equivalent:

(i) Whenever $x \operatorname{dom}_{W} z \operatorname{dom}_{W} y$ for some $z \in \Phi$, then either $z=x$ or $z=y$ (thus forming a cover of dominance);

(ii) There exists a $w \in W$ such that $w x \in D_{0}$ and $w y \in-D_{0}$.

Proof. Suppose that (i) is the case. Let $w$ be as in Proposition 4.11 above. First we show that then $w x \in D_{0}$. Suppose for a contradiction that $w x \notin D_{0}$, and let $z \in D(w x)$. Then Proposition 4.11 yields that $w y \in \Phi^{-}$and $(w y, z) \geq(w x, z) \geq 1$. Hence it is clear that $z \operatorname{dom}_{W} w y$. But this implies that $x \operatorname{dom}_{W} w^{-1} z \operatorname{dom}_{W} y$ with $x \neq w^{-1} z \neq y$, contradicting (i). Therefore $w x \in D_{0}$, as required. Exchanging the roles of $x$ and $-y$ we may deduce that $w y \in-D_{0}$.

Suppose that (ii) is the case and suppose for a contradiction that there exists some $z \in \Phi \backslash\{x, y\}$ such that $x \operatorname{dom}_{W} z \operatorname{dom}_{W} y$. Let $w \in W$ with $w x \in D_{0}$ and $w y \in-D_{0}$. If $w z \in \Phi^{+}$then Lemma 3.2(ii) yields that $w x \operatorname{dom}_{W} w z$, contradicting the fact that $w x \in D_{0}$. On the other hand, if $w z \in \Phi^{-}$, then parts (ii) and (iii) of Lemma 3.2 yield that $-w y \operatorname{dom}_{W}-w z \in \Phi^{+}$, contradicting the fact that $-w y \in D_{0}$.

Observe that applying Corollary 4.17 to arbitrary reflection subgroup $W^{\prime}$ of $W$ yields the following:

Corollary 4.18. Suppose that $W^{\prime}$ is a reflection subgroup of $W$ with $x$ and $y \in$ $\Phi\left(W^{\prime}\right)$ being distinct. The following are equivalent:

(i) Whenever $x \operatorname{dom}_{W^{\prime}} z \mathrm{dom}_{W^{\prime}} y$ for some $z \in \Phi\left(W^{\prime}\right)$, then either $z=x$ or $z=y$;

(ii) There exists a $w \in W^{\prime}$ such that $w x \in D_{W^{\prime}, 0}$ and $w y \in-D_{W^{\prime}, 0}$.

Definition 4.19. Suppose that $W^{\prime}$ is a reflection subgroup of $W$ and $x, y \in \Phi\left(W^{\prime}\right)$ satisfy both (i) and (ii) of Corollary 4.18. Then we say that the dominance between $x$ and $y$ is minimal with respect to $W^{\prime}$.

Proposition 4.20. Suppose that $x, y \in \Phi$ are distinct with $x \operatorname{dom}_{W} y$, and let $W^{\prime}$ be the dihedral reflection subgroup generated by $r_{x}$ and $r_{y}$. Then the dominance between $x$ and $y$ with respect to $W^{\prime}$ is minimal.

Proof. It follows from Corollary 3.3(ii) that $x \operatorname{dom}_{W^{\prime}} y$, and hence Lemma 3.4 yields that $W^{\prime}$ is infinite dihedral. Let $\Delta\left(W^{\prime}\right)=\{\alpha, \beta\}$. Then Proposition 3.17(i) yields that $D_{W^{\prime}, 0}=\{\alpha, \beta\}$.

On the other hand, it follows from Proposition 4.6 that there is some $w \in W^{\prime}$ such that

$$
\left\{\begin{array} { l } 
{ w x = a , } \\
{ w y = - b , }
\end{array} \text { or } \quad \left\{\begin{array}{l}
w x=b \\
w y=-a ;
\end{array}\right.\right.
$$


consequently Corollary 4.18 yields that the dominance between $x$ and $y$ with respect to $\left\langle\left\{r_{x}, r_{y}\right\}\right\rangle$ is minimal.

From this proposition we may deduce:

Proposition 4.21. Suppose that $x \in \Phi^{+}$with $D(x)=\left\{x_{1}, x_{2} \ldots, x_{m}\right\}$. For each $i \in\{1,2, \ldots, m\}$, set $W_{i}=\left\langle\left\{r_{x}, r_{x_{i}}\right\}\right\rangle$. Then $W_{i} \neq W_{j}$ whenever $i \neq j$.

Proof. For each $i \in\{1,2, \ldots, m\}$, set $\left\{s_{i}, t_{i}\right\}=S\left(W_{i}\right)$. Suppose for a contradiction that $W^{\prime}=W_{i}=W_{j}$ for some $i \neq j$. Then we may write $\{s, t\}=\left\{s_{i}, t_{i}\right\}=\left\{s_{j}, t_{j}\right\}$. Corollary 3.3(ii) yields that $x \operatorname{dom}_{W_{k}} x_{k}$ for all $k \in\{1,2, \ldots, m\}$, and since there is no nontrivial dominance in finite Coxeter groups, it follows that $W_{1}, W_{2}, \ldots, W_{m}$ are all infinite dihedral reflection subgroups. Hence it follows from Proposition 4.5.4 of [Björner and Brenti 2005] that $\left(\alpha_{s}, \alpha_{t}\right) \leq-1$. Set $c_{n}$ as in Proposition 3.5 for each $n \in \mathbb{Z}$. Since $x \operatorname{dom}_{W} x_{i}$ and $x \operatorname{dom}_{W} x_{j}$, Proposition 3.5(ii) yields that either

$$
\left\{\begin{array} { l } 
{ x = c _ { m } \alpha _ { s } + c _ { m + 1 } \alpha _ { t } , } \\
{ x _ { i } = c _ { m ^ { \prime } } \alpha _ { s } + c _ { m ^ { \prime } + 1 } \alpha _ { t } , } \\
{ x _ { j } = c _ { m ^ { \prime \prime } } \alpha _ { s } + c _ { m ^ { \prime \prime } + 1 } \alpha _ { t } , }
\end{array} \quad \text { or } \quad \left\{\begin{array}{l}
x=c_{m} \alpha_{s}+c_{m-1} \alpha_{t}, \\
x_{i}=c_{m^{\prime}} \alpha_{s}+c_{m^{\prime}-1} \alpha_{t}, \\
x_{j}=c_{m^{\prime \prime}} \alpha_{s}+c_{m^{\prime \prime}-1} \alpha_{t},
\end{array}\right.\right.
$$

for some distinct integers $m, m^{\prime}$ and $m^{\prime \prime}$. Observe that in either case $\left(x_{i}, x_{j}\right) \geq 1$, and therefore there will be (nontrivial) dominance between $x_{i}$ and $x_{j}$. Without loss of any generality, we may assume that $x \operatorname{dom}_{W} x_{i} \operatorname{dom}_{W} x_{j}$. Then

$$
x \operatorname{dom}_{W^{\prime}} x_{i} \operatorname{dom}_{W^{\prime}} x_{j}
$$

by Corollary 3.3(ii), contradicting Proposition 4.20.

We close this paper with an alternative characterization for the imaginary cone $Q$ when $\# \Delta<\infty$.

Proposition 4.22. If $\# \Delta<\infty$ then

$$
Q=\left\{w v \mid w \in W \text { and } v \in P \text { such that }(v, a) \leq 0 \text { for all } a \in \Phi^{+}\right\} .
$$

Proof. First we denote the set on the right-hand side of (4-6) by $Z$, and for each $b \in P$, define $\operatorname{Pos}(b)=\left\{c \in \Phi^{+} \mid(b, c)>0\right\}$. Recall that, under the assumption that $\# \Delta<\infty$, Lemma 4.4 yields that

$$
Q=\left\{v \in \bigcap_{w \in W} w P \mid(v, a) \leq 0 \text { for all but finitely many } a \in \Phi^{+}\right\} .
$$

Let $u \in Q$ be arbitrary. Since \# $\Delta<\infty$, it follows from Lemma 4.4 that $u \in P$. If $\operatorname{Pos}(u)=\varnothing$, then trivially $u \in Z$. Therefore we may assume that $\operatorname{Pos}(u) \neq \varnothing$, and proceed by induction on \# $\operatorname{Pos}(u)$ (this is only possible because $u \in Q$, and so $\# \operatorname{Pos}(u)<\infty)$. Let $a \in \Delta$ be chosen such that $(u, a)>0$. Then it can be readily 
checked that $\operatorname{Pos}\left(r_{a} u\right)=r_{a}(\operatorname{Pos}(u) \backslash\{a\})$. Thus the inductive hypothesis yields that $r_{a} u \in Z$. Clearly $Z$ is $W$-invariant, and so $u \in Z$, and hence $Q \subseteq Z$.

Conversely, if $x \in Z$, then $x=w v$ for some $w \in W$ and $v \in P$ such that $(v, a) \leq 0$ for all $a \in \Delta$. Lemma 4.5 yields that $v \in U^{*}$, and since $U^{*}$ is clearly $W$-invariant, it follows that $x \in U^{*}$. Suppose that $y \in \Phi^{+}$with $(x, y)>0$. Since $(x, y)=(w v, y)=\left(v, w^{-1} y\right)$, and since $(v, a) \leq 0$ for all $a \in \Phi^{+}$, it follows that $w^{-1} y \in \Phi^{-}$and thus $y \in N\left(w^{-1}\right)$. The finiteness of the set $N\left(w^{-1}\right)$ then implies that $x \in Q$, and hence $Z \subseteq Q$.

\section{Acknowledgments}

A few results in Section 4 of this paper are taken from the author's Ph.D. thesis [Fu 2010] and the author wishes to thank Professor R. B. Howlett for all his help and encouragement throughout the author's Ph.D. candidature. The author also wishes to thank Professors G. I. Lehrer and R. Zhang for supporting this work. Moreover, the author wishes to thank Professor Dyer for his penetrating insight and valuable suggestions. Finally, due gratitude must be paid to the referee of this paper, for her/his extraordinary patience and an exhaustive list of helpful suggestions; in particular, we thank the referee for pointing out to us the possibility of the converse of Theorem 4.13 .

\section{References}

[Björner and Brenti 2005] A. Björner and F. Brenti, Combinatorics of Coxeter groups, Graduate Texts in Mathematics 231, Springer, New York, 2005. MR 2006d:05001 Zbl 1110.05001

[Bourbaki 1968] N. Bourbaki, Éléments de mathématique, XXXIV: Groupes et algèbres de Lie, Actualités Scientifiques et Industrielles 1337, Hermann, Paris, 1968. MR 39 \#1590 Zbl 0186.33001

[Brink 1994] B. Brink, On root systems and automaticity of Coxeter groups, Ph.D. thesis, University of Sydney, 1994.

[Brink 1998] B. Brink, “The set of dominance-minimal roots”, J. Algebra 206:2 (1998), 371-412. MR 99k:20083 Zbl 0942.20020

[Brink and Howlett 1993] B. Brink and R. B. Howlett, "A finiteness property and an automatic structure for Coxeter groups", Math. Ann. 296:1 (1993), 179-190. MR 94d:20045 Zbl 0793.20036

[Deodhar 1982] V. V. Deodhar, "On the root system of a Coxeter group", Comm. Algebra 10:6 (1982), 611-630. MR 83j:20052a Zbl 0491.20032

[Dyer 1987] M. Dyer, Hecke algebras and reflections in Coxeter groups, Ph.D. thesis, University of Sydney, 1987.

[Dyer 1990] M. Dyer, "Reflection subgroups of Coxeter systems", J. Algebra 135:1 (1990), 57-73. MR 91j:20100 Zbl 0712.20026

[Dyer 1991] M. Dyer, "On the "Bruhat graph" of a Coxeter system", Compositio Math. 78:2 (1991), 185-191. MR 92c:20076 Zbl 0784.20019

[Dyer 2012] M. Dyer, "Imaginary cone and reflection subgroups of Coxeter groups", preprint, 2012. arXiv 1210.5206 
[Edgar 2009] T. Edgar, Dominance and regularity in Coxeter groups, Ph.D. thesis, University of Notre Dame, 2009, http://search.proquest.com/docview/851146656.

[Fu 2010] X. Fu, Root systems and reflection representations of Coxeter groups, Ph.D. thesis, University of Sydney, 2010, http://www.maths.usyd.edu.au/u/PG/Theses/X-Fu-2010_thesis.pdf.

[Fu 2012] X. Fu, "The dominance hierarchy in root systems of Coxeter groups", J. Algebra 366 (2012), 187-204. MR 2942649

[Hée 1990] J.-Y. Hée, "Le cône imaginaire d'une base de racines sur $\mathbb{R}$ ", unpublished, 1990.

[Hée 1993] J.-Y. Hée, Sur la torsion de Steinberg-Ree des groupes de Chevalley et des groupes de Kac-Moody, Ph.D. thesis, University of Paris-Sud, 1993.

[Hiller 1982] H. Hiller, Geometry of Coxeter groups, Research Notes in Mathematics 54, Pitman, Boston, MA, 1982. MR 83h:14045 Zbl 0483.57002

[Howlett 1996] R. B. Howlett, "Introduction to Coxeter groups", Lecture notes, Australian National University, 1996, http://www.maths.usyd.edu.au/res/Algebra/How/1997-6.ps.

[Howlett et al. 1997] R. B. Howlett, P. J. Rowley, and D. E. Taylor, "On outer automorphism groups of Coxeter groups", Manuscripta Math. 93:4 (1997), 499-513. MR 98j:20056 Zbl 0888.20023

[Humphreys 1990] J. E. Humphreys, Reflection groups and Coxeter groups, Cambridge Studies in Advanced Mathematics 29, Cambridge University Press, 1990. MR 92h:20002 Zbl 0725.20028

[Kac 1990] V. G. Kac, Infinite-dimensional Lie algebras, 3rd ed., Cambridge University Press, 1990. MR 92k:17038 Zbl 0716.17022

[Krammer 1994] D. Krammer, The conjugacy problem for Coxeter groups, Ph.D. thesis, Utrecht University, 1994, http://homepages.warwick.ac.uk/ masbal/index_files/me.pdf. Zbl 1176.20032

[Krammer 2009] D. Krammer, “The conjugacy problem for Coxeter groups”, Groups Geom. Dyn. 3:1 (2009), 71-171. MR 2010d:20047 Zbl 1176.20032

[Saunders 1991] C. Saunders, "The reflection representations of some Chevalley groups", J. Algebra 137:1 (1991), 145-165. MR 92f:20042 Zbl 0742.20016

Received January 26, 2012. Revised October 19, 2012.

XIANG FU

SCHOOL OF MATHEMATICS AND STATISTICS

UNIVERSITY OF SYDNEY

CARSLAW BUILDING (F07)

SYDNEY NSW 2006

Australia

x.fu@maths.usyd.edu.au 


\title{
PACIFIC JOURNAL OF MATHEMATICS
}

\author{
msp.org/pjm
}

Founded in 1951 by E. F. Beckenbach (1906-1982) and F. Wolf (1904-1989)

\section{EDITORS}

V. S. Varadarajan (Managing Editor)

Department of Mathematics

University of California

Los Angeles, CA 90095-1555

pacific@math.ucla.edu

Paul Balmer

Department of Mathematics

University of California

Los Angeles, CA 90095-1555

balmer@math.ucla.edu

Daryl Cooper

Department of Mathematics

University of California

Santa Barbara, CA 93106-3080 cooper@math.ucsb.edu

Jiang-Hua $\mathrm{Lu}$

Department of Mathematics

The University of Hong Kong

Pokfulam Rd., Hong Kong jhlu@maths.hku.hk
Don Blasius

Department of Mathematics University of California

Los Angeles, CA 90095-1555

blasius@math.ucla.edu

Robert Finn

Department of Mathematics Stanford University

Stanford, CA 94305-2125

finn@math.stanford.edu

Sorin Popa

Department of Mathematics

University of California

Los Angeles, CA 90095-1555

popa@math.ucla.edu

Paul Yang

Department of Mathematics

Princeton University

Princeton NJ 08544-1000

yang@math.princeton.edu

\section{PRODUCTION}

Silvio Levy, Scientific Editor, production@msp.org

\section{SUPPORTING INSTITUTIONS}

ACADEMIA SINICA, TAIPEI

CALIFORNIA INST. OF TECHNOLOGY

INST. DE MATEMÁTICA PURA E APLICADA

KEIO UNIVERSITY

MATH. SCIENCES RESEARCH INSTITUTE

NEW MEXICO STATE UNIV.

OREGON STATE UNIV.

\author{
STANFORD UNIVERSITY \\ UNIV. OF BRITISH COLUMBIA \\ UNIV. OF CALIFORNIA, BERKELEY \\ UNIV. OF CALIFORNIA, DAVIS \\ UNIV. OF CALIFORNIA, LOS ANGELES \\ UNIV. OF CALIFORNIA, RIVERSIDE \\ UNIV. OF CALIFORNIA, SAN DIEGO \\ UNIV. OF CALIF., SANTA BARBARA
}

\author{
Vyjayanthi Chari \\ Department of Mathematics \\ University of California \\ Riverside, CA 92521-0135 \\ chari@math.ucr.edu \\ Kefeng Liu \\ Department of Mathematics \\ University of California \\ Los Angeles, CA 90095-1555 \\ liu@math.ucla.edu \\ Jie Qing \\ Department of Mathematics \\ University of California \\ Santa Cruz, CA 95064 \\ qing@cats.ucsc.edu
}

These supporting institutions contribute to the cost of publication of this Journal, but they are not owners or publishers and have no responsibility for its contents or policies.

See inside back cover or msp.org/pjm for submission instructions.

The subscription price for 2013 is US \$400/year for the electronic version, and \$485/year for print and electronic.

Subscriptions, requests for back issues and changes of subscribers address should be sent to Pacific Journal of Mathematics, P.O. Box 4163, Berkeley, CA 94704-0163, U.S.A. The Pacific Journal of Mathematics is indexed by Mathematical Reviews, Zentralblatt MATH, PASCAL CNRS Index, Referativnyi Zhurnal, Current Mathematical Publications and the Science Citation Index.

The Pacific Journal of Mathematics (ISSN 0030-8730) at the University of California, c/o Department of Mathematics, 798 Evans Hall \#3840, Berkeley, CA 94720-3840, is published monthly except July and August. Periodical rate postage paid at Berkeley, CA 94704, and additional mailing offices. POSTMASTER: send address changes to Pacific Journal of Mathematics, P.O. Box 4163, Berkeley, CA 94704-0163.

PJM peer review and production are managed by EditFLOW ${ }^{\circledR}$ from Mathematical Sciences Publishers.

PUBLISHED BY

mathematical sciences publishers

nonprofit scientific publishing

http://msp.org/

(C) 2013 Mathematical Sciences Publishers 


\section{PACIFIC JOURNAL OF MATHEMATICS}

Volume $262 \quad$ No. $2 \quad$ April 2013

Certifying incompressibility of noninjective surfaces with scl

DANNY CALEGARI

Global well-posedness for the 3D rotating Navier-Stokes equations with highly oscillating initial data

QIONGLEI CHEN, CHANGXING MiAO and ZHIFEI ZHANG

Presenting Schur superalgebras

HOUSSEIN El TURKEY and JONATHAN R. KUJAWA

Classifying zeros of two-sided quaternionic polynomials and computing zeros of two-sided polynomials with complex coefficients

FENG LIANGGUI and ZHAO KAIMING

Coxeter groups, imaginary cones and dominance

XIANG FU

Semicontinuity of automorphism groups of strongly pseudoconvex domains: The low differentiability case

Robert E. Greene, Kang-Tae Kim, Steven G. Krantz and

AERYEONG SEO

Klein four-subgroups of Lie algebra automorphisms

JING-SONG HUANG and JUN YU

Fractal entropy of nonautonomous systems

Rui KuANG, WEN-ChiaO CHENG and BING LI

A GJMS construction for 2-tensors and the second variation of the total

$Q$-curvature

YoshiniKo MaTSUMOTO

Droplet condensation and isoperimetric towers

Matteo Novaga, Andrei Sobolevski and Eugene Stepanov

Brauer's height zero conjecture for metacyclic defect groups

BENJAMIN SAMBALE 